\title{
Note sur un élevage d'oies des Landes avec essais de production de foies gras à Madagascar
}

\author{
par H. SERRES, P. CAPITAINE, J. GILIBERT, \\ B. de REVIERS, J. J. RIBOT, P. DAYNES \\ et G. CHATILLON
}

\begin{abstract}
RESUME
La race doies landaises a été introduite à Madagascar en vue de la production de foie gras.

Les performances d'élevage sont analogues à celtes observées sous climat tempéré. La période de reproduction en est plus longue, décalée sans être en contre-saison. Le climat ne semble pas avoir d'influence défavorable sur les résultats du gavage qui sont très satisfaisants. L'étude du gavage d'oies locales a été menée sans succès et celui des oies métisses a donné des résultats intermédiaires. L'élevage de l'oie landaise est proposé à la vulgarisation.
\end{abstract}

\section{INTRODUCTION}

Les foies gras d'oies bénéficient sur le marché mondial d'une demande importante et de prix élevés. Or les deux principaux facteurs de production en sont le maiss et un travail manuel faiblement spécialisé de caractère saisonnier.

A Madagascar, dans certaines régions, le maïs est produit abondamment; les pratiques culturales sont souvent saisonnières (riziculture), et laissent un sous-emploi temporaire de la main-d'œuvre agricole; la main d'œuvre féminine est bon marché et fréquemment disponible; enfin l'élevage de l'oie locale pour la production de chair est développé.

Les carcasses d'oies grasses sont l'objet d'une demande certaine de la part des consommateurs malgaches et leur commercialisation ne doit présenter aucune difficulté.

Se sont donc posés les problèmes suivants : - Le gavage des oies locales a-t-il un intérêt?
- Le gavage d'oies landaises importées est-il réalisable?

- Quelles sont les possibilités de reproduction d'animaux importés?

- Le gavage d'oies landaises nées sur place et celui d'oies métisses sont-ils possibles et dans quelles conditions?

- Les foies produits ont-ils une qualité qui permet leur commercialisation à l'étranger ?

- Le prix de revient est-il compétitif ?

Un certain nombre de réponses ont été apportées à ces questions depuis 3 ans, elles font l'objet de la présente publication.

\section{PREMIERE PARTIE}

\section{ELEVAGE DES OIES LANDAISES AU C.R.Z. DE KIANJASOA}

\section{Le milieu}

Le C.R.Z. de Kianjasoa est situé dans le 
Moyen-Ouest de Madagascar (19 degrés latitude Sud 46,20 degrés longitude Ouest) où l'altitude est voisine de $900 \mathrm{~m}$.

Le climat y est de type tropical.

Les températures moyennes sont de 18,5 degrés en juillet, 24,5 degrés en novembre. Les minimums absolus du mois le plus chaud (janvier) sont 15,1 degrés et 32,9 degrés $C$, ceux du mois le plus froid (juillet) de 7 degrés $\mathrm{C}$ et 29,7 degrés $C$.

La pluviométrie annuelle est voisine de $1.600 \mathrm{~mm}$. Les précipitations surviennent d'octobre à mi-avril, habituellement le soir par périodes de quelques jours séparées par des périodes sèches de 2 à 3 jours, d'où d'importantes variations d'ambiance.

La saison sèche dure 6 mois, de la mi-avril au début d'octobre. Le degré hygrométrique moyen mensuel varie de 52 p. 100 en aoûtseptembre à 73 p. 100 en février.
Les pâturages sont abondants et de bonne qualité pendant la saison humide, moins bons et moins abondants pendant la saison sèche.

\section{L'élevage des reproducteurs}

\section{A) Origine de la population}

Les oies des Landes utilisées dans les essais ont été importées à l'âge de un jour du domaine expérimental d'Artiguères (Landes) dépendant de l'Institut National de la Recherche Agronomique.

Le premier lot importé est né en juin 1965 à Artiguères et fut utilisé en partie pour des essais comparatifs de gavage, en partie pour la constitution d'un troupeau de reproducteurs.

Le second lot, né en mars 1966, fut utilisé pour un essai comparatif des influences du maïs blanc et du maïs jaune sur la qualité des foies produits.

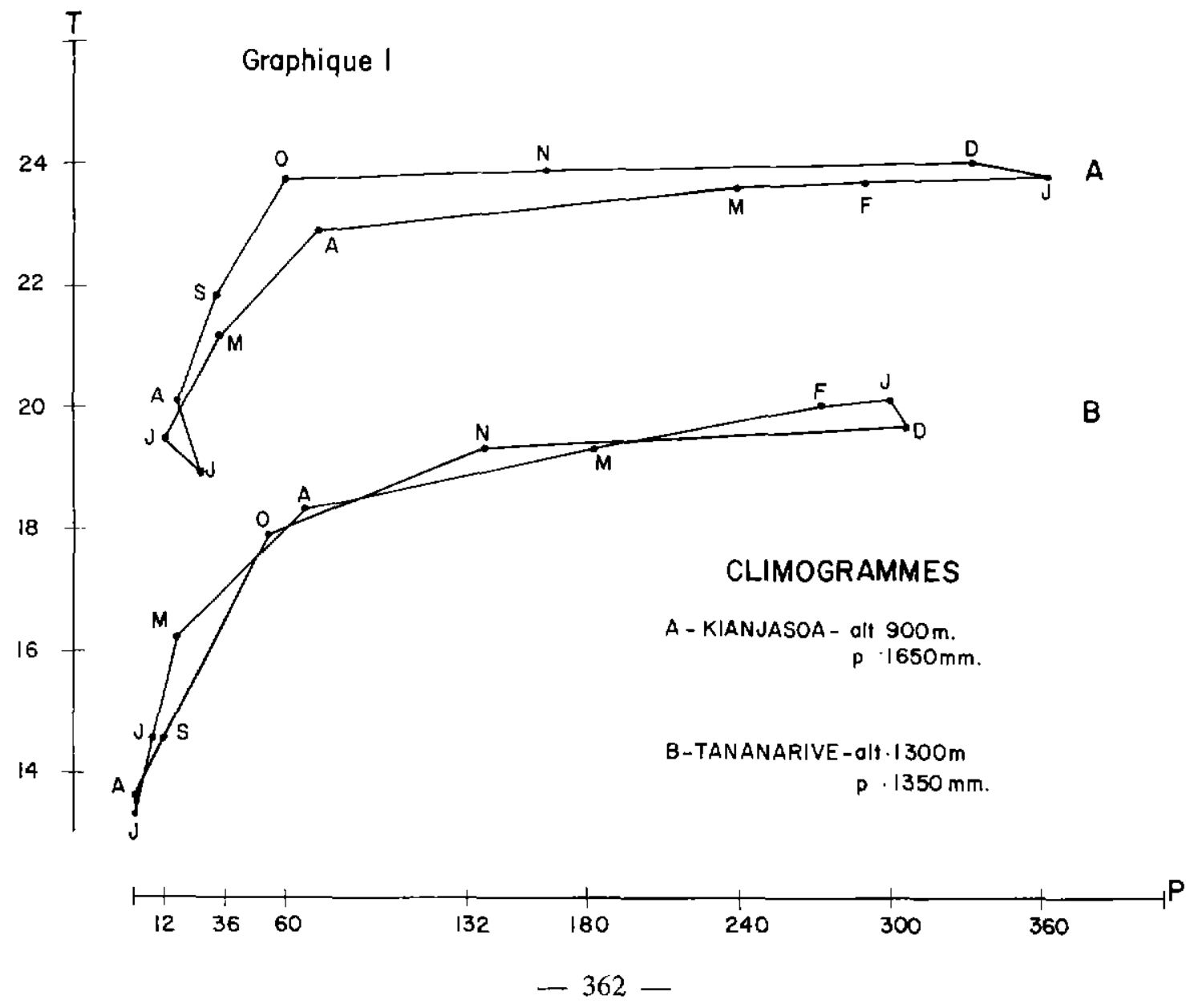




\section{B) Conduite de lélevage}

Les oiseaux disposent de loges grillagées bien aérées, installées dans des bâtiments de ferme déjà existants, à raison d'une loge pour dix. A l'époque de la ponte, des pondoirs garnis de foin sont installés dans les loges.

Les oies séjournent deux heures par jour sur des pâturages composés de Pueraria thumbergiana (Kudzu) soit, à défaut, de Stylosanthes gracilis. Elles consomment plus volontiers le Kudzu. Dans les loges, les reproducteurs ont de l'eau claire dans des abreuvoirs dont le fond est garni de sable; ils reçoivent au ratelier du Pueraria ou du Stylosanthes, ce dernier haché; ils ont à discrétion une provende composée de 50 p. 100 de son fin de riz et de 50 p. 100 d'un aliment complet "Oisons" dont la composition sera donnée au chapitre se rapportant à l'élevage des jeunes.

En période de copulation les animaux sont laissés en liberté toute la matinée autour d'une mare, l'accouplement n'ayant lieu qu'en milieu aquatique.

Aucun adulte n'est mort pendant l'essai.

\section{Reproduction des oisons}

\section{A) Reproduction et ponte}

Les premiers accouplements furent observés au début de février 1967. Les animaux avaient alors 11 mois. La ponte, commencée fin avril, se prolongea jusqu'à la fin octobre. La saison de ponte, d'une durée de 6 mois, fut donc plus longue que celles communément observées en France (4 mois $1 / 2$ ), et devait les années suivantes se déplacer vers la fin de l'année, par suite sans doute, du changement de rythme biologique dû au passage d'un hémisphère à l'autre. Le nombre total d'œufs produits, un millier, correspondait à plus de trente œufs par oie femelle dans la saison. Ce chiffre est à rapprocher de ceux indiqués par VUATRIN et d'autres auteurs qui estiment la production à 35 œufs la première année, 40 les deuxième et troisième et une trentaine au-delà.

La répartition des périodes de ponte est indiquée dans le graphique 2 où l'on remarque que la production présente 3 périodes séparées par 2 arrêts d'une semaine, l'un survenant 6 semaines après le début de la ponte, le second 6 semaines après le début du premier.

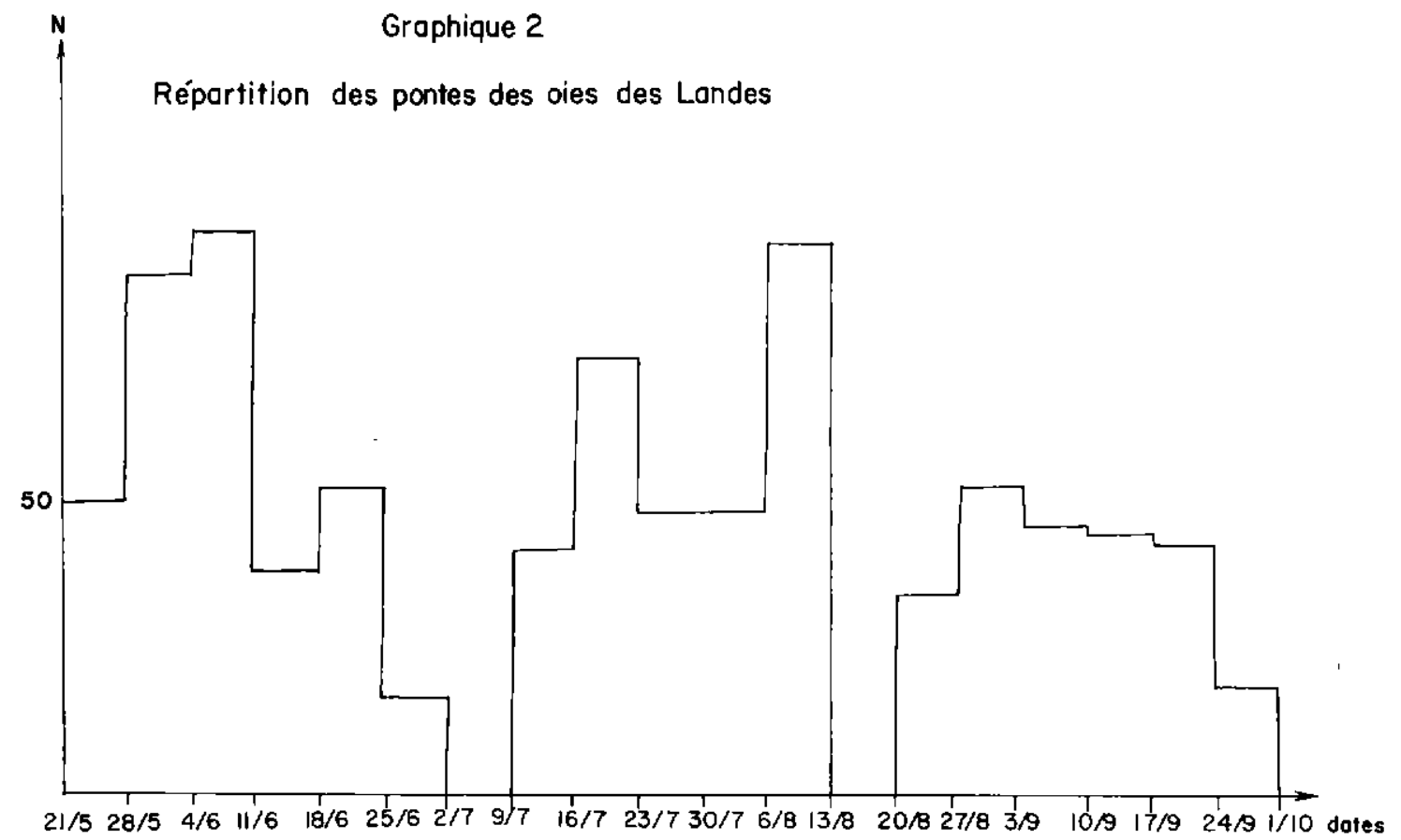


Le poids moyen des ceufs est de 174,6 \pm $11,3 \mathrm{~g} ; \mathrm{n}=810$ (extrêmes $140-210$ ).

La différence entre les poids des œufs à la mi-juin et à la fin octobre n'est pas significative.

\section{B) Accouvage}

L'incubation des œufs est faite dans des couveuses thermostatiques «La Nationale " fonctionnant au pétrole. La température est réglée à 38,3 degrés $\mathrm{C}$ à deux centimètres audessus du grillage des paniers. L'hygrométrie est à saturation.

Entre le $12^{\circ}$ et le $25^{\mathrm{e}}$ jour les cufs sont aérés $10 \mathrm{mn}$ par jour.

Du $15^{\mathrm{r}}$ au $25^{\mathrm{e}}$ jour, ils sont légèrement humectés quotidiennement par aspersion avec une eau tiède et propre.

Dans les conditions ci-dessus, les naissances se sont échelonnées de fin avril au début novembre, la durée d'incubation étant de 27 à 32 jours et le poids à la naissance de $93,2 \mathrm{~g} \pm$ $9,03(\mathrm{n}=350)$.

Il n'y avait aucune différence significative entre les poids de naissance de lots nés en juin et en octobr:

Le pourcentage des éclosions (cufs éclos pour 100 cufs mis dans les incubateurs) pour l'ensemble de la saison de ponte a été de $40 \mathrm{p}$. 100. Les chiffres variaient de 36,4 à $69,8 \mathrm{p}$. 100 selon les périodes, les résultats les meilleurs étant observés en septembre à la fin de la saison de ponte (cf. tableau 1).

\section{TABLEAU $N^{\circ} \mathrm{I}$}

\begin{tabular}{|c|c|c|c|c|c|}
\hline $\begin{array}{c}\text { Période de } \\
\text { ponte }\end{array}$ & $\begin{array}{c}16 / 07 \\
22 / 07\end{array}$ & $\begin{array}{c}04 / 08 \\
09 / 08\end{array}$ & $\begin{array}{c}22 / 08 \\
27 / 08\end{array}$ & $\begin{array}{l}01 / 09 \\
05 / 09\end{array}$ & $\begin{array}{c}10 / 09 \\
17 / 09\end{array}$ \\
\hline $\begin{array}{c}\text { Pourcentage } \\
\text { d'éclosions }\end{array}$ & 36,4 & 32,8 & 54,8 & 69,8 & 40,8 \\
\hline
\end{tabular}

Les normes admises en France en élevage fermier sont de 40 p. 100 et DELPECH et Collab. citent des variations allant de 37,6 à 47,6 p. 100; les résultats obtenus à Kianjasoa sont donc du même ordre que ceux observés en France.

\section{C) L'Elevage au premier âge}

Après l'éclosion, le jeune oison reste au moins 24 heures à jeun dans la couveuse. Il est ensuite mis sous éleveuse. Etant donné le matériel disponible et le rythme des naissances, les jeunes oisons sont conduits par lots d'une quinzaine. Ils disposent d'une aire à la température ambiante du local (entre 17 degrés $\mathrm{C}$ et 25 degrés $C$ ) où sont disposés auges et abreuvoirs; le tout sur litière paillée.

La température sous l'éleveuse est :

- la $1^{\text {re }}$ semaine de 35 - 38 degrés

- la $2^{\text {v }}$ semaine de $30-32$ degrés

- la $3^{4}$ semaine de $25-28$ degrés

La densité des animaux est de l'ordre de 8 par $\mathrm{m}^{2}$. Ils disposent d'un aliment complet, dont les composants sont fournis par le tableau $\mathrm{n}^{\circ} 2$ et dont la constitution brute est la suivante :

\section{TABLEAU 2}

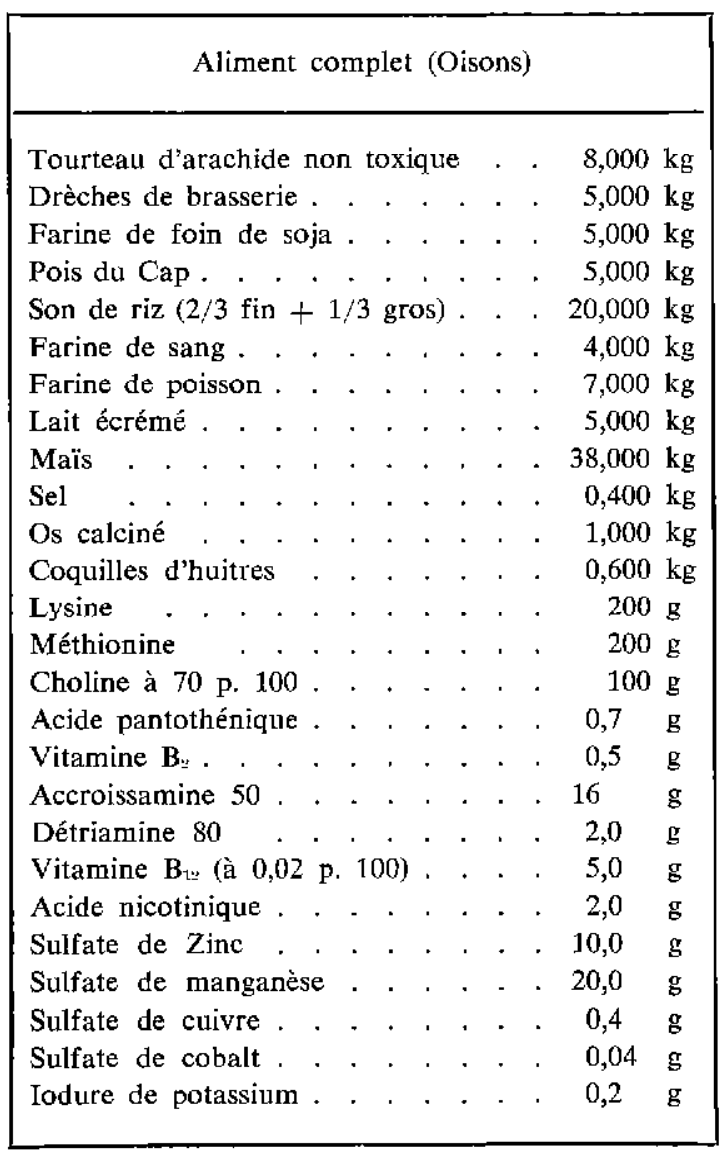




\begin{tabular}{|c|c|c|c|}
\hline \multirow{2}{*}{ Technique employëe } & \multirow{2}{*}{ Détermination } & \multicolumn{2}{|c|}{ Résultats pour $100 \mathrm{~g}$} \\
\hline & & $\begin{array}{c}\text { de prodult brut } \\
\text { (en g) }\end{array}$ & $\begin{array}{c}\text { de produit sec } \\
\text { (en g) }\end{array}$ \\
\hline & Composition & & \\
\hline Dessiccation i $103^{\circ} \mathrm{C}$ & Eau & 9,66 & - \\
\hline Incinération à $550^{\circ} \mathrm{C}$ & Matières munérales & 7,75 & 8,58 \\
\hline $\begin{array}{l}\text { Double extraction par } \\
1 \text { 'Ether Sulfurique }\end{array}$ & Matières grasses & 5,56 & 6,15 \\
\hline $\begin{array}{l}\text { Kjeldah1 } \\
(N . \text { Total } \times 6,25)\end{array}$ & Matiòres azotées & 20,23 & 22,39 \\
\hline $\begin{array}{l}\text { Selon léthode de } \\
\text { Weende }\end{array}$ & Cellulose órute & 4,66 & 5,16 \\
\hline Par diffêtrence & $\begin{array}{l}\text { Extractif non azoté } \\
\text { (Glucides, composés } \\
\text { pectiques, etc.) }\end{array}$ & 52,14 & 57,71 \\
\hline & $\begin{array}{l}\text { Dêterminations } \\
\text { complëmentaires }\end{array}$ & & \\
\hline $\begin{array}{l}\text { Méthode pondérale après } \\
\text { pêroxydation nitrique }\end{array}$ & Insoluble chlorhydrique & 1,84 & 2,04 \\
\hline Mêthode au Vanadate & Phosphore (en P) & 0,937 & 1,037 \\
\hline $\begin{array}{l}\text { Manganimétrie de } \\
1^{\prime} \text { oxalate }\end{array}$ & Calcium (en $\mathrm{Ca}_{a}$ ) & 1,046 & 1,158 \\
\hline
\end{tabular}

\section{D) L'Elevage au deuxième âge}

Après trois semaines les oisons sont logés sous abri léger, à la température ambiante (13 à 27 degrés $\mathrm{C}$ ), sur litière paillée, avec des abreuvoirs en permanence; ils sortent une ou deux heures par jour sur un pâturage de graminées (Chloris gayana).

Ils disposent à discrétion, en libre choix, de la provende " oisons " dont la composition est donnée au paragraphe précédent (tableau $\mathrm{n}^{\circ} 2$ ), de farine de maïs, et de Stylosanthes haché.

\section{Résultats zootechniques}

\section{A) Adaptation}

Les oies des Landes se sont parfaitement adaptées aux conditions d'élevage en vigueur au C.R.Z. de Kianjasoa, comme en attestent les résultats zootechniques.

\section{B) Fécondité}

Les résultats exposés au chapitre reproduction et ponte sont comparables à ceux obtenus en Europe.

\section{C) Croissance des jeunes}

Les différents lots ont eu des croissances très voisines. La courbe des croissances moyennes, établie pour l'ensemble de la population, est représentée par le graphique $n^{\circ} 3$. Cette courbe croît très rapidement jusqu'à la sixième semaine; elle s'infléchit de la $6^{*}$ à la $8^{\mathrm{C}}$ selon les lots; une certaine reprise de croissance se manifeste jusqu'à la douzième ou la treizième semaine. Puis les poids se stabilisent. Ces performances sont comparables à celles obtenues en Europe.

L'interprétation des variations en cours de croissance doit être celle fournie par PAVAUX et FAYET (4), pour les oisons des Landes.

\section{D) Croisement entre l'oie landaise et loie malgache}

Le croisement entre l'oie landaise et l'oie malgache n'a présenté aucune difficulté et s'est avéré fécond.

\section{Pathologie et mortalité}

A) Mortalité au premier âge : De la naissance à 3 semaines la mortalité a été de 


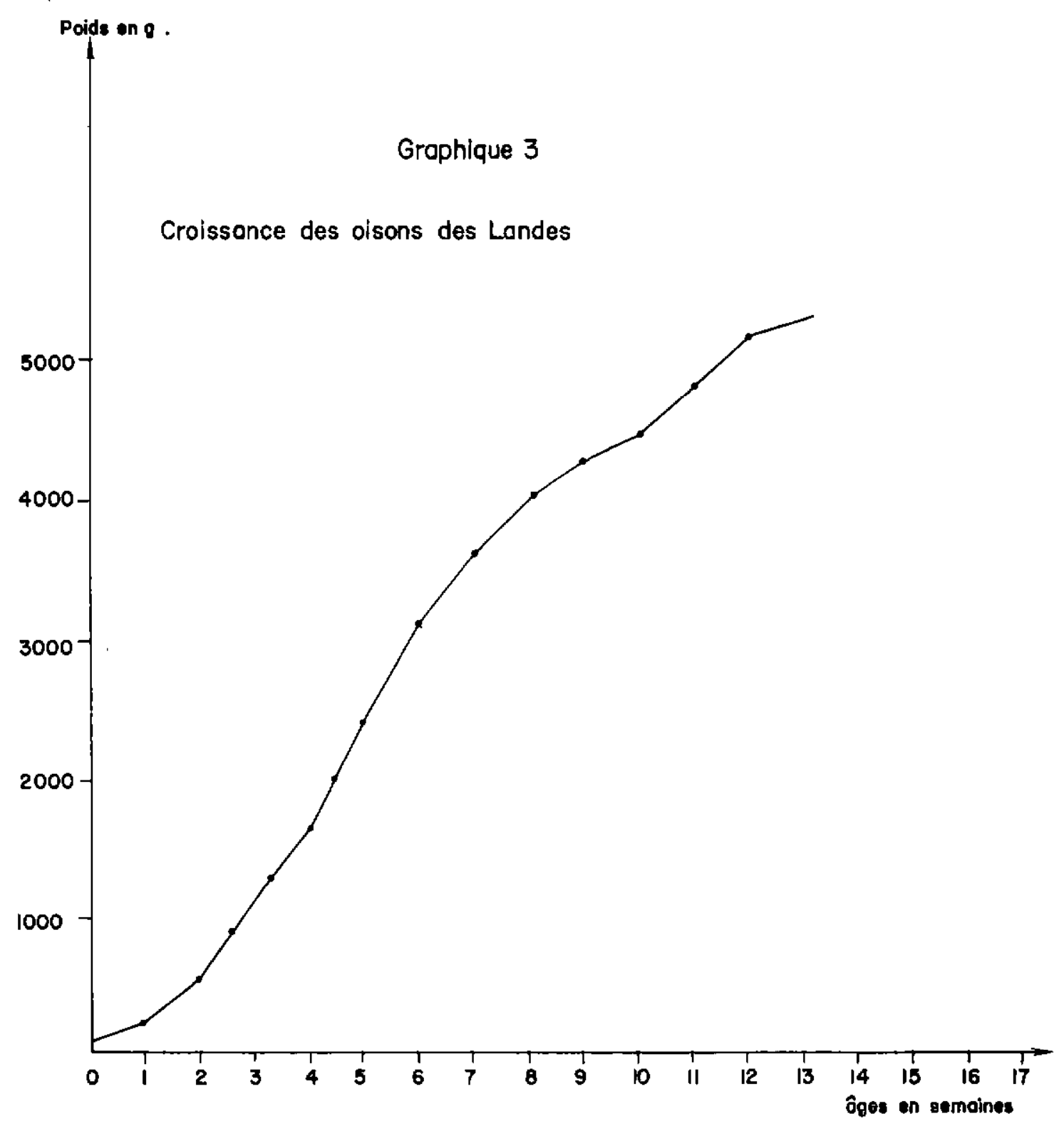

9,3 p. 100 en 1967 , imputable pour les $2 / 3$ à des irrésorptions du vitellus et pour $1 / 3$ à des écrasements ou étouffements sous l'éleveuse. Ces résultats sont du même ordre que ceux observés en 1966 sur les oisons importés, et légèrement supérieurs à ceux d'Europe.

B) Mortalité au deuxième âge: De trois semaines à trois mois et demi, 1,5 p. 100 des animaux nés sont morts, sans cause déterminée.

La mortalité sur les adultes, en dehors des gavages, est négligeable. Ces résultats confir- ment la parfaite adaptation des oies des Landes à Madagascar. La prophylaxie se limitait à une vaccination contre le choléra, lorsque les lots atteignaient l'âge de 3 mois.

On doit souligner que le choléra aviaire sévit toujours de façon endémique avec de vives poussées épizootiques à Madagascar. Un troupeau d'oisons non vaccinés en a été la victime. Cela impose la nécessité d'une prophylaxie, à la fois sanitaire et médicale, de cette maladie. 


\section{DEUXIEME PARTIE}

\section{PRODUCTION DE FOIES GRAS}

\section{Matériel et méthode}

\section{A) Prégavage}

Les animaux, parvenus à l'âge adulte, sont groupés en lots de gavage. Ils sortent une à deux heures par jour sur un pâturage de Kudzu.
(Pueraria thumbergiana). Cette légumineuse verte leur est également distribuée au ratelier. lls reçoivent par ailleurs un aliment de prégavage comprenant 60 p. 100 de maïs concassé, 20 p. 100 de farine de sang, 20 p. 100 de tourteau d'arachide, et dont la composition est fournie au tableau 4.

Cet aliment est distribué à vonlonté, avec consommation mesurée. Le prégavage dure 3 semaines.

\begin{tabular}{|c|c|c|}
\hline \multirow[b]{3}{*}{ Composition } & \multicolumn{2}{|c|}{ Résultats pour $100 \mathrm{~g}$} \\
\hline & $\begin{array}{c}\text { de produit brut } \\
(\text { en } g)\end{array}$ & $\begin{array}{l}\text { de produit sec } \\
\text { (en g) }\end{array}$ \\
\hline & & \\
\hline Eau & 10,34 & - \\
\hline Mattères minérales & 3,29 & 3,67 \\
\hline Matières grasses & 4,38 & 4,89 \\
\hline Mat1ères azotēes & 29,46 & 32,86 \\
\hline Ceilulose brute & 2,48 & 2,77 \\
\hline $\begin{array}{l}\text { Extractif non azot } \vec{e} \\
\text { (Glucldes, composés pectiques) }\end{array}$ & 50,05 & 55,81 \\
\hline Déterminations comolémentaires & & \\
\hline Insoluble chlorhydrique & 0,63 & 0,70 \\
\hline Phosphore (en P) & 0,328 & 0,366 \\
\hline Calctum (en $\mathrm{Ca}$ ) & 0,088 & 0,098 \\
\hline
\end{tabular}

Cet allment est distribué à volonté, avec consommation mesurée. Le prégavage dure 3 semaines.

\section{B) Gavage proprement dit}

Pendant cette période, les oies sont immobilisées en claustration complète, dans le plus grand calme, sur litière de paille. Le gavage a lieu trois fois par jour à sept heures, onze heures et dix-sept heures. Les premiers essais ont fait appel au gavage à main; par la suite, des gaveuses électriques ont été utilisées.

Le maïs est bouilli au moins $10 \mathrm{mn}$, légèrement salé, malaxé avec du saindoux pour faciliter la déglutition. L'examen biquotidien des animaux permet de déterminer le moment de l'abattage en fonction des critères classiques (engraissement, essouflement, apathie ...)

Lors de l'abattage, l'animal est d'abord pesé vivant puis pendu par les pattes et saigné à la carotide, plumé et pesé de nouveau. Le foie est alors extrait au scalpel, débarrassé de la vésicule et des gros canaux biliaires, puis pesé. Toutes ces opérations, du gavage à l'extraction du foie, ont vu leur résultat s'améliorer à mesure que le personnel acquérait la technicité nécessaire. Ce facteur humain s'est avéré très important.

\section{Gavage d'oies des Landes}

Des oies landaises ont été gavées au Laboratoire Central de l'Elevage de Tananarive.

On a d'abord utilisé des animaux nés à Artiguères, importés au cours des premiers jours de leur vie à Madagascar, puis élevés jusqu'à l'âge de 5 mois au Centre de Kianjasoa. 
Sur ces premières oies, on a pratiqué le gavage soit avec du maïs jaune soit avec du maïs blanc.

Par la suite on a pu gaver des oies landaises nées et élevées à Madagascar.

Les résultats obtenus au cours de ces essais (rassemblés aux trois premières colonnes du tableau 5) sont particulièrement satisfaisants pour la production des foies gras, puisque la moyenne générale sur 82 foies, de $770 \mathrm{~g}$, est excellente. La répartition en classes de poids est figurée au graphique $4: 49$ foies pèsent entre 600 et $1.000 \mathrm{~g}$ et font donc partie de la catégorie la plus recherchée; 15 sont au-dessus de $1.000 \mathrm{~g}$ et, bien qu'excellents, sont légèrement dépréciés au $\mathrm{kg}$ parce que trop gras; 14 enfin pèsent moins de $600 \mathrm{~g}$, dont deux seulement moins de $400 \mathrm{~g}$.

Il faut noter que les durées de gavage et les qualités de maïs consommé sont excessives. Cela s'explique par le manque de métier des gaveurs qui, craignant de léser les oies, ne poussaient pas suffisamment l'ingestion forcée.

Il en est résulté une prolongation de l'opération et une consommation finalement abusive.
Mais la technicité des opérateurs s'est progressivement améliorée. Les durées de gavage sont passées de 52 jours à 35 jours, ce qui se rapproche des durées pratiquées en Europe.

Les poids des foies obtenus avec le lot gavé au maïs blanc étaient plus faibles (moyenne $639 \mathrm{~g})$ que ceux obtenus avec un lot gavé simultanément pendant la même durée, avec la même quantité de maïs, par le même personnel, avec du maïs jaune (moyenne $861 \mathrm{~g}$ ). La différence n'est pas significative au seuil de 5 p. 100 . Les foies obtenus au maïs blanc présentaient une teinte jaune crème, tandis que ceux obtenus au maïs jaune étaient un peu plus foncés, plutôt jaune-brun. Mais de toute manière la teinte était toujours assez claire. Il ne paraissait pas, après cuisson, que la différence demeure sensible.

On soulignera que, sur les oies gavées au maïs jaune, les plus nombreuses, il a été recherché s'il y avait une corrélation entre le poids des oies au moment de leur sacrifice, et le poids des foies. Le résultat a été négatif.

La comparaison peut aussi être faite entre les résultats obtenus avec les oies importées et ceux fournis par les oies nées à Madagascar.

TABLEAU $\mathrm{H}^{\circ} \mathrm{V}$

(moyenne $\pm 1,96$ erreur standard)

\begin{tabular}{|c|c|c|c|c|}
\hline & $\begin{array}{c}\text { Landaises importées } \\
n=27\end{array}$ & $\begin{array}{c}\text { Landaises 1mportées } \\
\mathrm{n}=17\end{array}$ & $\begin{array}{c}\text { Landaises locales } \\
5-6 \text { mois } \\
n=38\end{array}$ & $\begin{array}{c}\text { Landaises lacales } \\
\begin{array}{c}3-4 \text { mois } \\
n=7\end{array}\end{array}$ \\
\hline Maís utilisë & Jaune & Blanc & Jaune & Jaune \\
\hline $\begin{array}{l}\text { Poids an fin de } \\
\text { gavage }(\mathrm{kg})\end{array}$ & $9,38 \pm 0,60$ & $8,92 \pm 0,45$ & $9,13 \pm 0,32$ & $7,4 \pm 0,31$ \\
\hline $\begin{array}{l}\text { Polds en début de } \\
\text { gavage (kg) }\end{array}$ & $4,96 \pm 0,28$ & $5,07 \pm 0,35$ & $4,70 \pm 0,18$ & $5,05 \pm 0,57$ \\
\hline Gain de poids $(\mathrm{kg})$ & $4,42 \pm 0,36$ & $3,85 \pm 0,41$ & $4,43 \pm 0,24$ & $2,35 \pm 0,39$ \\
\hline $\begin{array}{l}\text { Duree du gavage } \\
\text { (jours) }\end{array}$ & 51,5 & 52 & 42,3 & 18,7 \\
\hline Polds des foles (g) & $861 \pm 79,5$ & $639 \pm 90,7$ & $763 \pm 69,3$ & $681 \pm 165,6$ \\
\hline $\begin{array}{l}\text { Indice d'efficacité } \\
\text { économlque }(p \cdot 100)(x)\end{array}$ & 9,2 & 6,4 & 8,4 & 9,14 \\
\hline Maís consnmmé $\left(\mathrm{kg}_{\mathrm{g}}\right)$ & 54,3 & 54,5 & 41,4 & 18,7 \\
\hline
\end{tabular}

(x) L'indice d'efficactée ëconomique est le pourcentage du foie par rapport su poids te l'ole salgnée, plumêe, non vidée. 
Le tableau 5 montre qu'ils sont très proches et qu'en particulier pour les foies gras on obtient :

$$
\mathrm{m} \pm 1,96 \mathrm{sm}
$$

Oies importées (44) $775 \pm 60,7$ (g)

Oies locales (38) $763 \pm 69,3(\mathrm{~g})$

La différence n'est pas significative.

Pendant ces gavages, huit oies durent être abattues d'urgence pour accidents de gavage, mais néanmoins elles ont pu être récupérées, après saignée, pour consommation de la carcasse. Deux oies ont été trouvées mortes en fin de gavage et n'ont pu être récupérées.

Un essai complémentaire a porté sur 7 oies jeunes. Il est rapporté en quatrième colonne du tableau 5. Selon SZUMOWSKI et IVACHKIEVITCH, la possibilité de produire des foies gras, en mettant à l'engraissement des oies âgées de 2 à 4 mois, est certaine.

Une tentative a été faite sur un petit groupe de huit oies landaises de trois mois et demi.

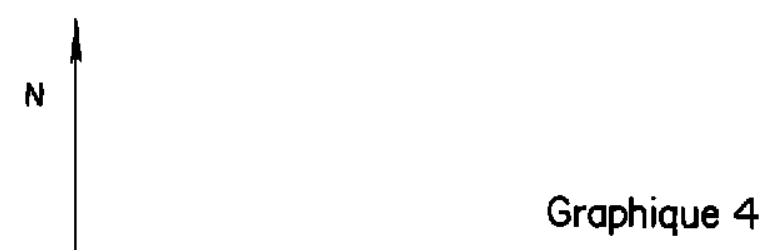

Répartition des poids de 82 foies d'oies landaises
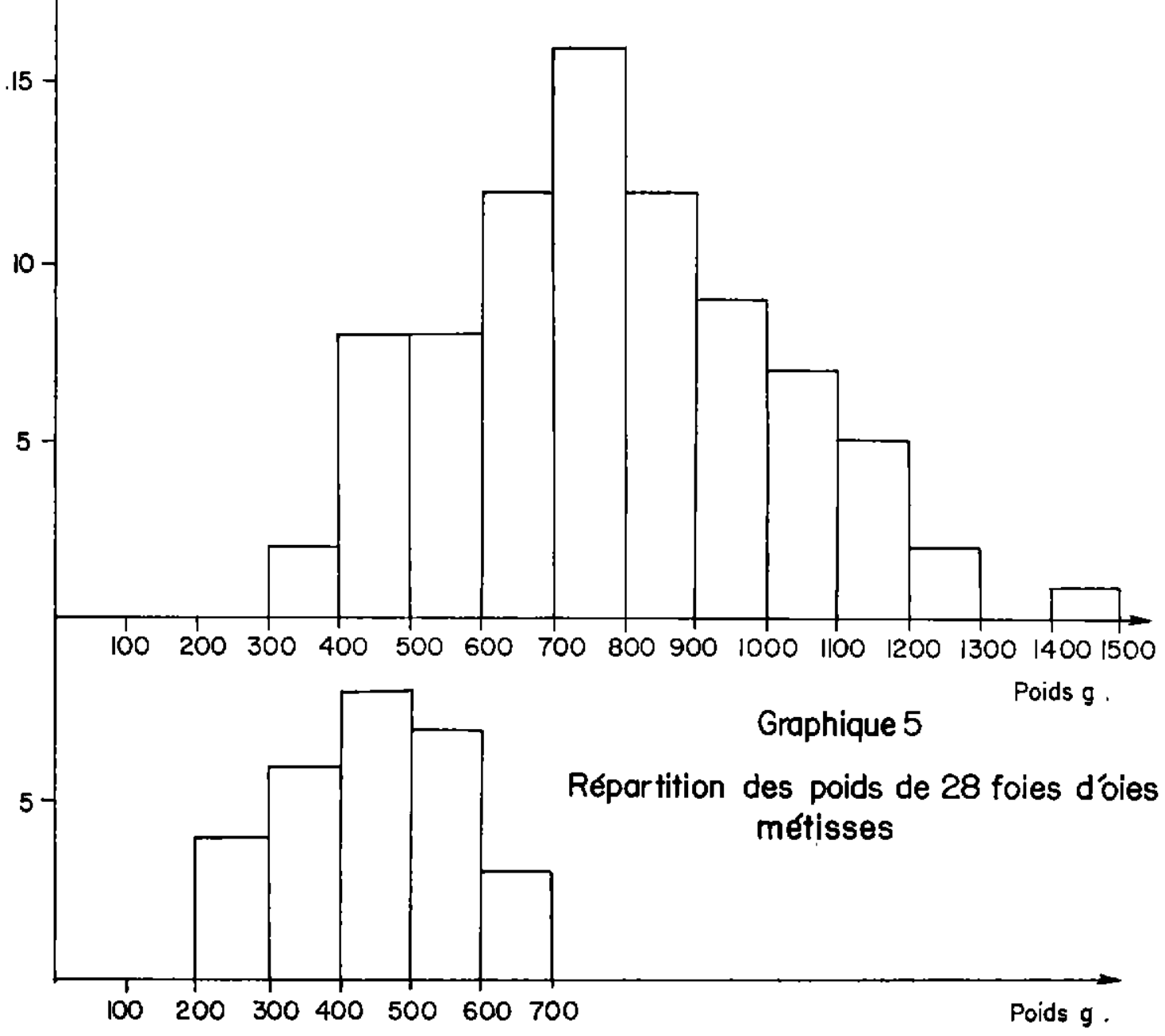
L'essai a été pénible pour les animaux et l'une des oies est morte en cours de gavage. Les résultats pour les sept survivantes sont néanmoins très satisfaisants puisqu'on a obtenu une moyenne de $681 \mathrm{~g}$ pour les foies, après 19 jours de gavage seulement.

Il faut noter qu'un deuxième essai a dû être interrompu par suite d'accidents de gavage plus nombreux.

L'utilisation d'animaux jeunes ne paraît pouvoir se pratiquer que si l'on possède un personnel particulièrement attentif. D'autre part, la moyenne de poids des foies est plus faible et le revenu consécutivement inférieur; surtout pour les foies pesant moins de $600 \mathrm{~g}$, plus nombreux, qui sont à la fois plus légers et de moindre valeur au $\mathrm{kg}$. Il n'est pas sûr que le gain de deux mois d'élevage à l'herbe puisse compenser et le manque à gagner et les pertes plus nombreuses.

Pour terminer, nous ferons remarquer que plusieurs essais de gavage ont été réalisés en pleine saison chaude de l'été austral. Les oies paraissaient plus essoufflées, mais les performances de production n'ont nullement été altérées. Physiologiquement elles ont donc bien supporté les températures élevées, de l'ordre de 30 degré $\mathrm{C}$, auxquelles elles étaient soumises pendant la journée, alors que la nuit le thermomètre ne descendait pas au-dessous de 25 degrés dans leur local.

\section{Gavage d'oies malgaches}

Douze oies malgaches, achetées au marché ont été gavées sans que soit fait le prégavage. Les animaux se sont assez bien comportés: une oie est morte le $33^{e}$ jour, mais son foie a été néanmoins pesé; les autres ont été sacrifiées le $35^{\mathrm{e}}$ jour.

Les résultats sont rassemblés au tableau 6 .

On remarquera que les poids des foies sont très faibles, puisque leur moyenne n'atteint pas $115 \mathrm{~g}$. Presque tous étaient restés totalement rouges et aucun n'avait pris la teinte jaune uniforme recherchée.

L'augmentation du poids des oies est demeurée également modeste (moins de $1,5 \mathrm{~kg}$ ), bien qu'elles aient en moyenne consommé $25 \mathrm{~kg}$ de maïs.
Ces résultats sont particulièrement décevants et condamnent l'utilisation de l'oie malgache pour la production de foies gras.

TABLEAU $N * V I$

\begin{tabular}{|l|c|}
\hline & $\begin{array}{c}\text { Oies (12) } \\
\text { malgaches }\end{array}$ \\
\hline $\begin{array}{l}\text { Polds des oies au début } \\
\text { du gavage (kg) }\end{array}$ & 4,4 \\
\hline $\begin{array}{l}\text { Poids des oies en f1n } \\
\text { de gavage (kg) }\end{array}$ & 5,86 \\
\hline Gain de poids (kg) & 1,46 \\
\hline Durée du gavage (jours) & 35 \\
\hline Polds des foles (g) & $114,5 \pm 44,6$ \\
\hline $\begin{array}{l}\text { Indice d'efficacité } \\
\text { éconounque (p,100) }\end{array}$ & 1,9 \\
\hline Maiss consomé (kg) & 25 \\
\hline
\end{tabular}

\section{Gavage d'oies métisses de première génération}

Afin d'accroître les possibilités de production d'oies à gaver, on peut penser à l'amélioration des oies malgaches par croisement avec des jars de race landaise.

Ce croisement, nous l'avons dit, ne présente pas de difficultés quant à la reproduction.

Les oisons s'élèvent très bien, soit séparés, soit mélangés aux oisons des Landes.

Deux lots d'oies métisses, issues du croisement de première génération (malgache $\times$ Landes) ont été gavés, après un prégavage. L'un des lots, de 14 animaux, était composé d'oies de 5 mois. Il a été traité en même temps que les oies des Landes, à un moment où la technicité des gaveurs n'était pas suffisamment rodée, ce qui explique un gavage long (48 jours) et une importante consommation de maïs.

Les résultats sont groupés au tableau 7.

$$
\left(\mathrm{m}+1,96 \mathrm{~s}_{\mathrm{m}}\right)
$$

Les foies obtenus étaient d'aspect le plus souvent moyen, certains étaient franchement jaunes, d'autres marbrés. Mais, surtout, les poids obtenus étaient insuffisants pour les faire classer en première qualité (un seul foie de 
TABLEAU $\mathrm{N}^{\circ}$ VII

$\left(\mathrm{m}+1,96 \mathrm{~s}_{\mathrm{m}}\right)$

\begin{tabular}{|c|c|c|}
\hline & $\begin{array}{c}\text { Métisses de } 5 \text { à } 6 \text { mols } \\
n=14\end{array}$ & $\begin{array}{c}\text { Mét1sses de } 3 \text { à } 4 \text { mo1s } \\
\mathrm{n}=14\end{array}$ \\
\hline $\begin{array}{l}\text { Poids des ofes au début } \\
\text { du gavage }(\mathrm{kg})\end{array}$ & $4,75 \pm 0,31$ & $4,70 \pm 1,01$ \\
\hline $\begin{array}{l}\text { Poidg des oles en } \mathrm{f} \text { in } \\
\text { de gavage (kg) }\end{array}$ & $B, 82 \pm 0,39$ & $7,54 \pm 0,44$ \\
\hline Wain de poids (kg) & $4,07 \pm 0,67$ & $2,84 \pm 0,53$ \\
\hline Durée de gavage (jours) & 48,5 & 20 \\
\hline Polds des foles $(\mathrm{g})$ & $444,6 \pm 65$ & $475 \pm 67$ \\
\hline $\begin{array}{l}\text { Indtce d'efficacité } \\
\text { êconomlque (p.100) }\end{array}$ & 5,2 & 6,4 \\
\hline MaIs consorméé(kg) & 44,1 & 20,0 \\
\hline
\end{tabular}

plus de $600 \mathrm{~g}$ ). A $444 \mathrm{~g}$ de moyenne, on ne peut obtenir une rémunération élevée par la commercialisation des foies gras.

Pour la prise de poids de l'oie, par contre, on a gagné $4 \mathrm{~kg}$, ce qui se rapproche de ce que l'on obtient avec l'oie des Landes, tout en demeurant légèrement en retrait.

Les oies jeunes, gavées plus intensément, ont consommé près de $17 \mathrm{~kg}$ de maïs en 20 jours. Les poids moyens des foies gras $(475 \mathrm{~g})$ et leur aspect étaient comparables à ceux des oies adultes.

La répartition en classes des foies d'oies métisses est représentée au graphique 5, ce qui permet de voir le décalage par rapport aux foies obtenus avec les oies des Landes (graphique 4).

Il ne semble pas que les oies métisses de première génération, bien que donnant des résultats plus proches de ceux de l'oie des Landes que de ceux de l'oie Malgache, soient capables de fournir par gavage des foies gras de qualité, de façon régulière.

Comme l'élevage de l'oie des Landes s'est avéré facile à Madagascar, il vaut mieux que l'éleveur s'oriente vers la race pure landaise.

\section{CONCLUSION}

Le gavage des oies landaises donne à Madagascar des résultats analogues à ceux obtenus en Europe.
Le gavage des oies malgaches ne présente aucun intérêt : ces animaux n'ont pas d'aptitude à la dégénérescence graisseuse du foie, leurs indices de consommation sont trop élevés.

Le gavage d'oies métisses de première génération est moins intéressant que le gavage d'oies landaises de pure race, ses résultats sont moins réguliers et les indices de consommation plus élevés. Il pourrait présenter de l'intérêt si les oies landaises ne se reproduisaient pas normalement à Madagascar, ce qui n'est pas le cas.

Le gavage précoce d'animaux jeunes et en fin de croissance mériterait une étude plus approfondie car les résultats obtenus, sur un effectif réduit et à une période de l'année défavorable, demandent à être confirmés.

\section{TROISIEME PARTIE}

\section{TRAITEMENT DES FOIES APRES ABATTAGE}

\section{Expéditions}

La production des foies à Madagascar ne sera pleinement rentabilisée que si les foies sont exportés frais.

La voie aérienne fut la première à laquelle on pensa, mais le transport en container se révéla bien trop onéreux et en raison d'un retard au cours du voyage, l'une des expéditions fut entièrement perdue. 
L'expérience a montré que des foies, emballés dans des feuilles d'alliages inoxydables d'aluminium dès leur récolte et rapidement congelés en armoire frigorifique à $-40^{\circ} \mathrm{C}$ ou sur azote liquide, pouvaient être conservés pendant des temps variant entre une semaine et 3 mois sans perdre leurs qualités organoleptiques, la seule précaution consistant à les décongeler lentement pendant 24 heures en chambre froide à $+4^{\circ} \mathrm{C} /+5^{\circ} \mathrm{C}$ et à les traiter dès leur sortie de cette dernière.

Pour les envois par avion, les foies congelés emballés sont mis dans des boîtes en carton ondulé fort, doublées intérieurement de plaques de polychlorure de vinyl expansé de $4 \mathrm{~cm}$ d'épaisseur.

Le remplissage des boîtes est achevé avec de la neige carbonique.

Dans un tel emballage, tous les envois par avion de Tananarive vers Paris sont arrivés en excellent état.

Aucun envoi par la voie maritime n'a été fait, mais comme des foies conservés 3 mois à $-30^{\circ} \mathrm{C}$ et expédiés ensuite par avion sont arrivés en excellent état, on peut très bien envisager de faire des expéditions par bateau en cale frigorifique.

Des envois, par chemin de fer, de foies congelés ont été faits en juillet, sans incidents, en messagerie rapide de Paris à Terrasson en Dordogne.

\section{Qualités organoleptiques}

La valeur marchande d'un foie gras dépend d'une qualité objective, le poids, de qualités subjectives telles que la couleur, le grain, l'onctuosité et la saveur.

Les foies de 800 - 900 grammes sont les plus prisés, trop gros ils sont généralement trop gras et perdent une partie de leur graisse à la cuisson, trop petits, ils se prêtent mal aux fabrications de foie entier.

Les foies ocre jaune, ocre jaune rosé, sont les plus prisés, quant à la saveur, elle est très subjective.

Désirant connaître la valeur gastronomique des foies produits à Madagascar, des foies au début frais, ensuite congelés furent expédiés à Paris et traités par deux fabricants de haute renommée, M. GUIOT, Directeur des Etablissements BATTENDIER à Paris et un conserveur, M. FAVREAU à Terrasson, Dordogne.

Leurs expertises sont concordantes.

Les qualités des foies frais ou congelés reçus de Madagascar sont identiques à celles des foies achetés dans les Landes; couleur, grain onctuosité.

La conservation à basse température n'altère ni la couleur, si, bien entendu, la feuille d'aluminium est bien plaquée contre le foie, ni le grain, ni l'onctuosité.

Le jugement d'un des lots de 1968 qui comprenait 11 foies pour un poids total de $9,755 \mathrm{~kg}$, fut le suivant:

- 4 foies pesant respectivement, 1.200 , $1.050,900$ et $1.100 \mathrm{~g}$, avaient des qualités comparables à ceux des Landes, bien qu'un peu gros et étaient classés $1^{\text {er }}$ choix.

- 4 autres de 560, 600, 925 et $900 \mathrm{~g}$, un peu moins gras que les précédents, pouvaient être classés en $1^{\text {ter }}$ choix.

- 3 autres de $1.040,740$ et $740 \mathrm{~g}$ auraient été de bonne qualité si les oies avaient été mieux saignées.

Le jugement du lot de 1969 qui comprenait 19 foies d'un poids total de $14,580 \mathrm{~kg}$, était encore plus favorable puisque 12 étaient classés en $1^{\mathrm{er}}$ choix $(11,065 \mathrm{~kg}), 4$ en $2^{\circ}$ choix $(2,115 \mathrm{~kg}), 3$ en $3^{\mathrm{e}}$ choix $(1,400 \mathrm{~kg}$ dont $0,800 \mathrm{~kg}$ de déchets), ces derniers présentaient des lésions d'éclatement dues à un gavage trop avancé et des défauts de saignée.

Il est impossible de distinguer après cuisson au naturel et au porto ou après mise en boîtes, les foies congelés des foies frais.

Les tableaux $\mathbf{n}^{\circ 4} 8,9$ et 10 donnent le détail des expertises faites sur 3 lots de foies; le premier a été transporté en frais sur glace, les deux autres congelés.

Les deux premiers lots ont été traités en terrines ou en plats cuisinés.

Avec le dernier, les foies ont été mis dans des boîtes non vernies de 1/12 (100 g environ), 
sans autre assaisonnement que du sel, du poivre et des truffes. Les boites, après sertissage, ont été mises à l'autoclave à $100^{\circ}$ pendant 2 heures $1 / 2$ et refroidies dès la sortie.

A l'ouverture des boîtes échantillons, on note que la corrosion n'est pas plus importante qu'avec des foies d'origine européenne.

\section{CONCLUSIONS}

Les qualités techniques et organoleptiques des foies gras obtenus à Madagascar sont très comparables à celles des foies des Landes.

La congélation des foies à $-30^{\circ} /-40^{\circ} \mathrm{C}$ et leur conservation à $-30^{\circ} \mathrm{C}$ ne modific en rien leurs qualités.

TABLEAU $N^{\circ}$ VIII

Foles frais - ler lot expẹdiê sous glace

\begin{tabular}{|c|c|c|c|c|c|c|}
\hline Matricule & $\begin{array}{l}\text { Polds } \\
\text { frais }\end{array}$ & $\begin{array}{l}\text { Poids } \\
\text { cult. }\end{array}$ & Qualité frais & $\begin{array}{l}\text { Mode de } \\
\text { préparation }\end{array}$ & Qualit té cuit & Classement \\
\hline 1.319 & 600 & 480 & $\begin{array}{l}\text { Bonne qualité } \\
\text { grain un peu gros }\end{array}$ & Porto & Bon goût & 2ẽme Cholx \\
\hline 1.320 & 1.200 & 790 & $\begin{array}{l}\text { Bon aspect } \\
\text { Bon grain }\end{array}$ & Porto & $\begin{array}{l}\text { Bonne qualité } \\
\text { Manque un peu de } \\
\text { parfum }\end{array}$ & $\begin{array}{l}\text { ler Choix } \\
\text { Landes }\end{array}$ \\
\hline 1.323 & 740 & 690 & $\begin{array}{l}\text { Bonne qualité } \\
\text { Mal saigné }\end{array}$ & Naturel & $\begin{array}{l}\text { Un peu foncé } \\
\text { Bon goût }\end{array}$ & 2ème Choix \\
\hline 1.324 & 1.050 & 740 & $\begin{array}{l}\text { Excellent grain } \\
\text { Bon goût }\end{array}$ & Porto & $\begin{array}{l}\text { Excellent } \\
\text { Onctueux }\end{array}$ & $\begin{array}{l}\text { ler Cholx } \\
\text { Landes }\end{array}$ \\
\hline 1.326 & 900 & 800 & $\begin{array}{l}\text { Bon grain } \\
\text { Bonne couleur } \\
\text { Bon goût }\end{array}$ & Naturel & Très satisfalsant & $\begin{array}{l}\text { ler Choix } \\
\text { Landes }\end{array}$ \\
\hline 1.338 & 1.100 & 900 & $\begin{array}{l}\text { Exce1lent grain } \\
\text { Bonne couleur } \\
\text { Onctueux }\end{array}$ & Naturel & $\begin{array}{l}\text { Excellent grain } \\
\text { Très onctueux } \\
\text { Très parfumé }\end{array}$ & $\begin{array}{l}\text { ler Cholx } \\
\text { Landes }\end{array}$ \\
\hline 1.341 & 740 & 610 & $\begin{array}{l}\text { Bon grain un peu } \\
\text { foncê - mal salgné }\end{array}$ & Naturel & $\begin{array}{l}\text { Foncé } \\
\text { Assez bon goût }\end{array}$ & 2ème Choix \\
\hline 1.343 & 610 & 560 & $\begin{array}{l}\text { Grain un peu gros } \\
\text { Un peu sec }\end{array}$ & Naturel & $\begin{array}{l}\text { Restê foncé } \\
\text { mais onctueux }\end{array}$ & 2ème Chalx \\
\hline 1.381 & 1.040 & 800 & $\begin{array}{l}\text { Bon grain - bonne } \\
\text { couleur mais mal } \\
\text { saigné }\end{array}$ & Porto & Exce1lent & $\begin{array}{l}\text { ler Cholx } \\
\text { Landes }\end{array}$ \\
\hline $\begin{array}{l}2.508 \\
\text { Métis }\end{array}$ & 530 & 530 & $\begin{array}{l}\text { Mal saigné - goût } \\
\text { un peu fort } \\
\text { Qualité Budapest }\end{array}$ & Terrine & $\begin{array}{l}\text { Satisfaisant sans } \\
\text { plus - goût de } \\
\text { terroir un peu fort }\end{array}$ & $\begin{array}{l}\text { 2ème Choix } \\
\text { Hongrie }\end{array}$ \\
\hline
\end{tabular}

\section{QUATRIEME PARTIE}

\section{ETUDE PREVISIONNELLE DU COUT DE L'OIE GAVEE}

\section{Description du projet}

A la suite des expériences d'élevage et d'engraissement d'oies des Landes réalisées au Centre de Recherches de Kianjasoa et au Laboratoire Central de l'Elevage à Tananarive, des données techniques valables pour cette région ont pu être déterminées. Elles apparaissent encourageantes.

Leur intérêt est de permettre dès à présent une étude économique prévisionnelle sur les possibilités de production de foies gras en vue de l'exportation et sur la compétitivité que l'on peut attendre de Madagascar en ce domaine.

Pour pouvoir tirer profit des expériences que nous avons décrites et pour des raisons que nous justifierons plus loin, nous avons choisi l'étude de la production de foies gras dans le 
TABLEAU $\mathbb{N}^{0}$ IX

Lot $n^{\circ} 2$ - Foles congelês - conservês au plus 15 jours - traítés en plats cuisinês

\begin{tabular}{|c|c|c|c|c|c|c|}
\hline Matricule & $\begin{array}{l}\text { Polds } \\
\text { frais }\end{array}$ & $\begin{array}{l}\text { Poids } \\
\text { cult. }\end{array}$ & Qualitế frais & $\begin{array}{c}\text { Mode de } \\
\text { préparation }\end{array}$ & Qualitê cult & Classement \\
\hline 1.339 & 1.130 & 1.040 & $\begin{array}{l}\text { Bon grain } \\
\text { Lêgêrement rosê } \\
\text { Bon goût }\end{array}$ & Roulade & $\begin{array}{l}\text { Garde son graín } \\
\text { un peu plat mais } \\
\text { bonne qualité }\end{array}$ & $1 / 2$ \\
\hline 1.349 & 800 & 800 & $\begin{array}{l}\text { Bonne qualitê } \\
\text { Gra1n un peu gros } \\
\text { Bon goût }\end{array}$ & Boîte & $\begin{array}{l}\text { Bon goût } \\
\text { Onctueux }\end{array}$ & 2 \\
\hline 1.357 & 630 & 610 & $\begin{array}{l}\text { Bon grain un peu } \\
\text { gros - jaune }\end{array}$ & $\begin{array}{l}\text { Naturel } \\
\text { au porto }\end{array}$ & $\begin{array}{l}\text { Garde son grain } \\
\text { Onctueux } \\
\text { Excellent }\end{array}$ & ler - Landes \\
\hline 1.358 & 650 & 480 & $\begin{array}{l}\text { Bon grain } \\
\text { Bonne couleur }\end{array}$ & $\begin{array}{l}\text { Naturel } \\
\text { au porto }\end{array}$ & Bon goût & 1er - Landes \\
\hline 1.371 & 1.040 & 800 & $\begin{array}{l}\text { Grain très fin } \\
\text { Belle couleur } \\
\text { Un peu fort }\end{array}$ & $\begin{array}{l}\text { Petit pain } \\
\text { en bofte }\end{array}$ & $\begin{array}{l}\text { Conserve son } \\
\text { goût } \\
\text { Excellent }\end{array}$ & ler - Landes \\
\hline 1.399 & 360 & 360 & $\begin{array}{l}\text { Bonne consistance } \\
\text { Un peu foncé } \\
\text { Goût Hongrie }\end{array}$ & Terrine & $\begin{array}{l}\text { Goût prononcê } \\
\text { Un peu granuleux } \\
\text { et sec }\end{array}$ & 2ème Choix \\
\hline 3.988 & 820 & 610 & $\begin{array}{l}\text { Grain excellent } \\
\text { Onctueux }\end{array}$ & $\begin{array}{l}\text { Foie nature } \\
\text { Porto }\end{array}$ & $\begin{array}{l}\text { Légèrement rosé } \\
\text { Un peu foncé } \\
\text { Bon goût quolque } \\
\text { un peu fort }\end{array}$ & 2ème Choix \\
\hline $\begin{array}{l}1.344 \\
\text { Métis }\end{array}$ & 410 & 410 & $\begin{array}{l}\text { Bonne consistance } \\
\text { Un peu foncé } \\
\text { Goût oriental }\end{array}$ & Terrine & $\begin{array}{l}\text { Un peu faure } \\
\text { Onctueux } \\
\text { Bon goût } \\
\text { Taches défectueu- } \\
\text { ses fancées dues } \\
\text { à une mauyaise } \\
\text { saignêe }\end{array}$ & 2ème Choix \\
\hline $\begin{array}{l}2.530 \\
\text { Métis }\end{array}$ & 430 & 430 & $\begin{array}{l}\text { Bonne conststance } \\
\text { Un peu trop jaune }\end{array}$ & Terrine & $\begin{array}{l}\text { Très jaune } \\
\text { Taches foncées }\end{array}$ & 2ème Chotx \\
\hline
\end{tabular}

cadre d'une unité de production rationnellement organisée et de dimensions telles qu’elle puisse rentabiliser convenablement ses moyens de production et s'insérer dans des structures déjà existantes.

C'est ainsi qu'il est apparu souhaitable de concevoir ce projet dans le cadre du développement du Centre National Avicole de Madagascar qui offre une situation, une organisation et des moyens tout à fait favorables à l'implantation d'une telle unité. Cette unité de production, de taille malgré tout restreinte, devrait permettre de mieux rentabiliser certains équipements du Centre National Avicole. Pour cette raison et là où c'était le cas, nous avons négligé le coût d'utilisation de ces équipements qui, de toutes façons, auraient représenté peu de chose dans le bilan final (exemple: utilisation de l'atelier à provende pour préparer les aliments pour oies). Bien entendu, si cette unité type devait être multipliée, il y aurait lieu de prendre ces facteurs en considération.

Avant de donner les caractéristiques techniques de ce projet, il importe de souligner que certaines des options choisies s'écartent de celles qui avaient été expérimentées au Centre de Recherches de Kianjasoa soit parce que les dimensions de l'unité que nous avons retenues les justifiaient manifestement, soit parce que les ressources offertes au Centre National Avicole le permettaient.

C'est en ce sens que cette étude est prospective et doit être considérée comme prévisionnelle.

Nous donnerons d'abord les caractéristiques techniques de l'unité choisie, puis nous passerons à l'analyse financière. 
TABLEAU $\mathrm{N}^{*} \mathrm{X}$

Lot $\mathrm{n}^{*} 3$ - Foles congelés - conservés 3 mols à $-30^{\circ} \mathrm{C}$ - Expertise conserverie

\begin{tabular}{|c|c|c|c|c|c|c|}
\hline Matricule & $\begin{array}{l}\text { Poids a la } \\
\text { congélation } \\
\text { (en g) }\end{array}$ & $\begin{array}{l}\text { Poids après } \\
\text { décongélation } \\
\text { (en } g \text { ) }\end{array}$ & $\begin{array}{l}\text { Qualités après } \\
\text { décongélation }\end{array}$ & Class ement & $\begin{array}{l}\text { Emplo1 } \\
\text { possible }\end{array}$ & $\begin{array}{l}\text { Qualités après traltetnent } \\
\text { à l'ouverture de la bolte }\end{array}$ \\
\hline 1.575 & 765 & 750 & $\begin{array}{l}\text { Bel aspect-ocre } \\
\text { clair-ferme grain } \\
\text { fln-quelques } \\
\text { taches de sang }\end{array}$ & 1er Choix & foie entier & $\begin{array}{l}\text { Teneur en graisse } 25 p \cdot 100 \\
\text { présentation moyenne } \\
\text { saveur agréable }\end{array}$ \\
\hline 1.567 & 725 & 725 & $\begin{array}{l}\text { Ocre jaune } \\
\text { Grain très fin }\end{array}$ & ler Choix & fole entier & $\begin{array}{l}\text { Teneur en graísse } 5 p \cdot 100 \\
\text { belle présentation } \\
\text { saveur agréable }\end{array}$ \\
\hline 1.561 & 740 & 720 & $\begin{array}{l}\text { Bel aspect-ocre } \\
\text { rose-Brain fin } \\
\text { quelques taches } \\
\text { de sang }\end{array}$ & 1er Choix & foie entier & $\begin{array}{l}\text { Teneur en graisse environ } \\
\text { 20p.100-Be1fe présentation } \\
\text { saveur agréable } \\
\text { grain moyen }\end{array}$ \\
\hline 1.571 & $4 B S$ & 470 & $\begin{array}{l}\text { Bel aspect ocre } \\
\text { Jaune - Mou - } \\
\text { grain fin }\end{array}$ & ler Choix & fole entier & $\begin{array}{l}\text { Teneur en graísse 5p.100 } \\
\text { Très beau grain fin. Très } \\
\text { belle prësentation. Belle } \\
\text { couleur. Saveur agréable }\end{array}$ \\
\hline SN 1 & 630 & 605 & $\begin{array}{l}\text { Bel aspect-ocre } \\
\text { Jaune - ferme - } \\
\text { grain fin }\end{array}$ & ler Cholx & fole entier & $\begin{array}{l}\text { Teneur en graisse } 5 p .100 \text {. } \\
\text { grain moyen-Belle couleur } \\
\text { Saveur agréable }\end{array}$ \\
\hline SN 2 & 420 & 375 & $\begin{array}{l}\text { Foie très mou- } \\
\text { Mal saigné }\end{array}$ & 3è Choix & $\begin{array}{l}\text { crème ou } \\
\text { mousse de } \\
\text { fole }\end{array}$ & Pas tralté \\
\hline 1.588 & 420 & 405 & $\begin{array}{l}\text { Bel aspect-ocre } \\
\text { jaune-mou - } \\
\text { grain fin }\end{array}$ & 1er Choix & & $\begin{array}{l}\text { Teneur en graisse lop.loo } \\
\text { grain fin-Belle prêsenta- } \\
\text { tion-Saveur agréable }\end{array}$ \\
\hline $\mathrm{SN} 3$ & 480 & 465 & $\left\{\begin{array}{l}\text { Aspect nolrâtre } \\
\text { en surface proba- } \\
\text { blement feuille } \\
\text { d'aluminfun pas } \\
\text { adhérente à la } \\
\text { surface du fole } \\
\text { intérieur ocre } \\
\text { clair-ferme- } \\
\text { grain fin }\end{array}\right.$ & 2è Cholx & $\begin{array}{l}\text { rouleau } \\
\text { mousse ou } \\
\text { crème }\end{array}$ & $\begin{array}{l}\text { Après êpluchage- } \\
\text { Galn moyen } \\
\text { Bonne saveur }\end{array}$ \\
\hline SN 4 & 480 & 455 & $\begin{array}{l}\text { Mou-éclaté sur le } \\
\text { côté intérieux du } \\
\text { lobe central }\end{array}$ & $3 e ̄ \quad$ Choix & $\begin{array}{l}\text { rouleau } \\
\text { mousse }\end{array}$ & $\begin{array}{l}\text { Pas traité - Impropre à } \\
\text { être traité }\end{array}$ \\
\hline 1.510 & 600 & 500 & $\begin{array}{l}\text { Foie éclaté pré- } \\
\text { sentant des zones } \\
\text { lnutilisables - } \\
\text { ma1 salgné ou ga- } \\
\text { vage trop poussé }\end{array}$ & $3 e \ddot{C}$ Choix & mousse & Pas traltë \\
\hline
\end{tabular}

L'unité de production comprend 160 oies dont 20 p. 100 de mâles. Elle se divise en 4 parquets de reproduction, de 40 oies chacun. La description technique, de même que l'analyse des coûts de production, est décomposée en 4 phases qui représentent des ateliers distincts et presque indépendants.

1. Production de l'oisillon d'un jour et entretien du troupeau des reproducteurs.

2. Production de l'oisillon d'un jour à un mois.
3. Production de l'oison d'une mois à quatre mois.

4. Gavage.

A) Données et hypothèses techniques pour la production d'oisons d'un jour

\section{Parquets de reproduction}

4 parquets de reproduction, chacun comprenant :

- un hangar de ponte : toiture de tôles - 
piliers et charpente en bois enduit - sol cimenté - parois grillagées - surface couverte : $300 \mathrm{~m}^{2}$ - abreuvoirs, rateliers, nids de ponte.

- une aire de parcours clôturée de $300 \mathrm{~m}^{2}$ - Accès à un bassin d'eau.

\section{Incubateur}

Incubateur mixte

Capacité 2.600 œufs de poule

Eclosoirs incorporés

Consommation électrique $9 \mathrm{kwh}$

Bâtiment d'incubation : il n'est pas nécessaire de le prévoir dans le cadre du Centre National Avicole.

\section{Main-d'seuvre}

Pour les 4 parquets de reproduction, 12 mois par an:

- 2 ouvriers agricoles spécialisés (OS1)

- 1 manœuvre sans qualification.

4. Hypothèses techniques d'élevage

Provende «Oies " préparée au Centre National Avicole . . . . . . 18 Fmg/kg Consommation quotidienne de provende par animal $280 \mathrm{~g}$

Coût de verdure supposé négligeable. Taux annuel de remplacement . . .30 p. 100

Reproducteurs féconds à 2 ans

Taux de ponte d'une femelle adulte . . . . . 35 cufs Taux d'éclosion. . . .50 p. 100 Animaux de réforme . . $1.000 \mathrm{Fmg}$

B) Données et hypothèses pour l'élevage d'un jour à un mois

1. Ensemble de 3 oisonnières construites en dur

$30 \mathrm{~m}^{2}$ par oisonnière

Sol cimenté

Couverture de tôles

5 à 8 oisillons par $\mathrm{m}^{2}$

Abreuvoirs et trémies à provende.

2. Ensemble de 3 éleveuses

Eleveuses à gaz
Capacité 500 à 700 poussins (200 oisillons)

Consommation de gaz $=270 \mathrm{Fmg} /$ jour $/$ éleveuse.

\section{Main-d'auvre}

1 ouvrier agricole spécialisé (OS1) pendant 6 mois pour l'ensemble des oisonnières.

\section{Hypothèses techniques d'élevage}

5 à 8 oisillons $/ \mathrm{m}^{2}$

Mortalité : 6 p. 100

Provende " oisillons" préparée au Centre National Avicole : $25 \mathrm{Fmg} / \mathrm{kg}$

Consommation quotidienne par oisillon: $150 \mathrm{~g}$.

C) Données et hypothèses techniques pour l'élevage d'un mois à quatre mois

\section{Bâtiments d'élevage}

4 parquets pouvant contenir 200 oisons chacun.

Chaque parquet comprend:

- Hangar léger de $80 \mathrm{~m}^{2}$ : couverture de tôles - sol cimenté - parois grillagées abreuvoirs, rateliers, etc. - litière paillée. - Aire de parcours clôturée : $200 \mathrm{~m}^{2}$.

\section{Main-d'ouvre}

Pour l'ensemble des 4 parquets : 2 ouvriers agricoles spécialisés (OS1) pendant 6 mois.

\section{Prairies temporaires}

Chaque parquet dispose de 5 ha de prairies temporaires à réinstaller tous les 4 ans. Les bâtiments d'élevage ont été prévus suffisamment vastes pour qu'il ne soit pas nécessaire de faire sortir les animaux au pâturage. On fera donc de l'affouragement en vert. Ceci est applicable également aux parquets de reproducteurs qui profitent en fait des mêmes pâturages. Dans le cadre du Centre National Avicole, il pourrait être concevable de faire des cultures de légumineuses et même de Pennisetum ou de Tripsacum si ceux-ci sont appréciés par les oies.

Dans ce cas, les surfaces seraient à revoir en fonction des rendements possibles. 


\section{Matériel agricole}

Le Centre National Avicole dispose déjà d'un tracteur de $60 \mathrm{CV}$ avec remorque. et d'une faucheuse, également sous utilisés, et qui seront donc généralement disponibles.

Par contre il faut prévoir un hache-paille.

\section{Hypothèses techniques d'élevage}

Durée de l'élevage par oison : 90 jours

Durée de la saison d'élevage : 8 mois Effectif maximal présent par parquet: 350 à 400 oisons

Mortalité : 1 p. 100

Provende « oison " préparée au Centre National Avicole à $18 \mathrm{Fmg} / \mathrm{kg}$

Consommation quotidienne par animal : $250 \mathrm{~g}$.

\section{D) Données et hypothèses techniques conceruant le gavage}

\section{Bâtiments}

1 bâtiment en dur de $170 \mathrm{~m}^{2}(5 \mathrm{~m} \times 34 \mathrm{~m})$

Sol cimenté

Couverture en tôle

400 loges individuelles où les oies sont maintenues en claustration.

\section{Matériel}

5 gavoirs électriques montés sur chariot et permettant de gaver 1 oie à la minute Capacité de gavage par gaveur: 70 oies Nombre de bandes de gavage : 6

Capacité de gavage par saison et par gaveur: 420 oies.

1 Congélateur pour conserver les foies Capacité 6001

Consommation quotidienne $4 \mathrm{kwh}$

1 Réfrigérateur pour conserver les carcasses d'oies

Capacité 3001

Consommation quotidienne $2,5 \mathrm{kwh}$

1 Réchaud à gaz.

\section{Main-d'auvre}

5 gaveurs, ouvriers agricoles spécialısés (OS1) pendant 6 mois.

\section{Hypothèses techniques}

Maïs nécessaire au gavage d'une oie . . . . . . . . . . $30 \mathrm{~kg}$

Saindoux nécessaire par oie . . $300 \mathrm{~g}$

\section{Etude économique ( $\left.{ }^{1}\right)$}

A) Cô̂t de production de l'oison d'un jour

1. Coût des investissements

- Hangar de ponte :

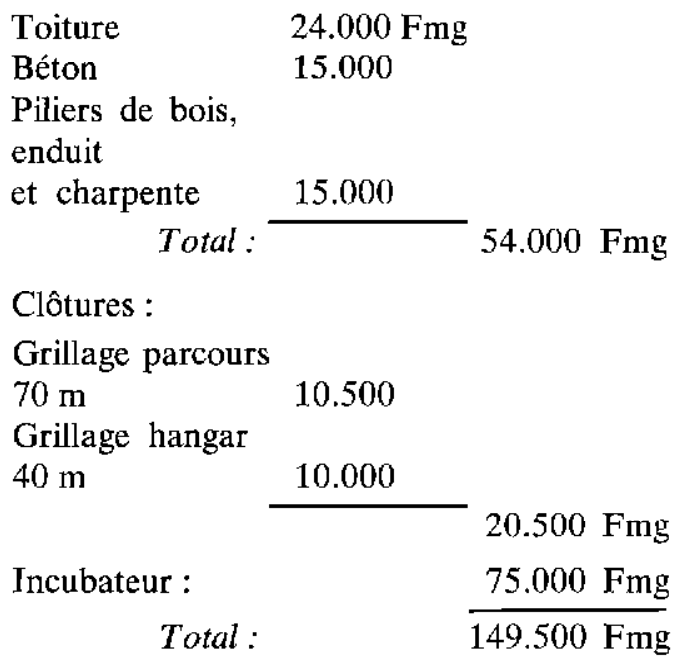

2. Charges annuelles

- Dépenses d'investissement :

$\begin{array}{lll}\text { Hangar de ponte } & 3.974 & \text { Fmg } \\ \text { Clôtures } & 4.736 & \\ \text { Incubateur } & 6.742 & \end{array}$

- Dépenses d'exploitation :

Main-d'cuvre $\quad 47.520$

Charges sociales $\quad 3.564$

Provende $\quad 117.734$

Entretien incuba-

teur (2p. 100$) \quad 1.500$

Entretien hangar

de ponte

(2 p. 100) $\quad 1.080$

Electricité $\quad 4.725$

Divers $\quad 5.000$

- Imprévus 10 p. 100 :

196.575 Fmg

Total : $19.657 \mathrm{Fmg}$ $216.232 \mathrm{Fmg}$

(1) Les coûts d'investissement et les charges sont rapportées à un parquet de 40 reproducteurs, c'est-àdire au quart de l'unité de production. 
3. Effectifs commercialisables

Oisons

Oisons de remplacement

Oisons commercialisables
4. Coût moyen de production de l'oison entre un jour et un mois

266 Fmg

C) Coût de production de l'oison d'un mois à quatre mois

4. Coût moyen de production de l'oison d'un jour

1. Cout des investissements

394 Fmg — Bâtiment d'élevage :

Toiture

64.000 Fmg

Remarque: Il n'a pas été tenu compte des Béton 40.000

animaux de remplacement d'un coût devant équilibrer la valeur de réforme des animaux remplacés.

Piliers

en bois enduit

et charpente

$\frac{40.000}{144.000 \mathrm{Fmg}}$

B) Coût de production de l'oison d'un jour et un mois

- Clôtures :

Grillage

1. Coût des investissements

Oisonnière $\quad 112.500 \mathrm{Fmg}$

Eleveuse

parcours $55 \mathrm{~m} \quad 8.250$

Grillage

bâtiment $36 \mathrm{~m} \quad 8.820$

et inverseur $\quad 23.200$

- Prairies temporaires :

17.070 Fmg

- Mat. agricole (hache-paille) :

25.000 Fmg

Total :

364.570 Fmg

2. Charges annuelles

- Dépenses d'investissement :

Bât. d’élevage $10.598 \mathrm{Fmg}$

Clôtures $\quad 3.943$

Prairies tempor. $\quad 51.515$

- Dépenses d'exploitation :

Main-d'œuvre $\quad 23.040$

Charges sociales $\quad 1.728$

Provende 208.575

Entr. bât. d'élevage

(2 p. 100) $\quad 2.880$

Imprévus 10 p. 100 :

124.718 Fmg

(1 p. 100) $\quad 1.125$

Divers $\quad 5.000$

12.472 Fmg Entr. mat. agricole

(5 p. 100) $\quad 1.250$

Divers $\quad 10.000$

316.611 Fmg

- Imprévus :

$31.661 \mathrm{Fmg}$

Total :

de pertes soit

348.272 Fmg 
3. Effectifs commercialisables

$515-1$ p. 100 soit 510 oisons

4. Coût moyen de production de loison d'un mois a quatre mois

\section{D) Coût du gavage}

1. Coût des investissements

Bât. de gavage $212.000 \mathrm{Fmg}$

Réfrigérateur $\quad 21.500$

Congélateur $\quad 36.250$

Loges pour oies $\quad 75.000$

Gavoir électr. $\quad 40.000$

Total : $\quad 384.750 \mathrm{Fmg}$

2. Charges annuelles

- Dépenses d'investissement:

$\begin{array}{lc}\text { Bâtiment } & 15.603 \text { Fmg } \\ \text { Réfrigérateur } & 2.651 \\ \text { Congélateur } & 4.470 \\ \text { Gavoir } & 4.932 \\ \text { Loges pour oies } & 5.520\end{array}$

- Dépenses d'exploitation:

Main-d'œuvre $\quad 43.200$

Charges sociales $\quad 3.240$

$\mathrm{Gaz} \quad 15.000$

Electricité $\quad 8.500$

Entretien bât. et

loges (1 p.100) $\quad 2.870$

Entretien matériel

électr. (3 p. 100) 5.528

Maïs $\quad 153.000$

Divers $\quad 5.000$

- Imprévus

10 p. 100 :

Total :

269.514 Fmg

$26.951 \mathrm{Fmg}$

296.465 Fmg

3. Effectifs commercialisables

510 - 5 p. 100 soit 485

4. Coût moyen du gavage par oie gavée

611 Fmg
E) Récapitulation de l'étude prévisionnelle de la production de foies gras pour une unité technique de 160 oies

L'unité de production est supposée s'insérer dans le Centre National Avicole et jouir des aménagements existant déjà sur le Centre, notamment en ce qui concerne l'atelier à provende, le bâtiment des incubateurs, le groupe électrogène et le matériel agricole. Le contrôle et la direction exercés par le Directeur du Centre pourraient également s'étendre à cette unité de production d'oies.

- Cô̂t des investissements, terrains exclus 4.478.000 Fmg

- Superficie nécessaire parcours et bâtiments environ 0,3 ha

Prairies temporaires 20 ha

- Main-d'euvre

2 ouvriers agricoles spécialisés (OS1) et 1 manouvre pendant 12 mois. 1 ouvrier agricole spécialisé (SO1) pendant 6 mois (juin à décembre).

2 ouvriers agricoles spécialisés (0S1) pendant 8 mois (juillet à mars).

5 ouvriers agricoles spécialisés (OS1), gaveurs, pendant 6 mois (octobre à avril).

Il semble nécessaire de prévoir en plus un commandeur (OS2) responsable tout au long de l'année de la bonne marche de l'entreprise. $\mathrm{Ce}$ commandeur sera compris dans les états suivants.

- Charges annuelles d'exploitation: répartition par poste

Main-d'œuvre $\quad 619.200$ soit 15 p. 100

Aliments $\quad 2.159 .840$ soit 53 p. 100

Autres $\quad 1.312 .190$ soit 32 p. 100 
- Production annuelle de foies gras

1940 unités soit environ $1.358 \mathrm{~kg}$ de foies gras.

- Prix de revient moyen par foie produit

2.109 Fmg

Ce coût est légèrement supérieur à celui que l'on obtiendrait en additionnant les coûts séparés des phases successives (1954) en raison de l'adjonction d'un commandeur et des pertes d'animaux.

F) Appréciation de la rentabilité de la production de foies gras

En raison de l'incertitude relative au prix de vente des foies gras, il s'agit là d'un calcul hypothétique mais qui semble néanmoins raisonnable :

\section{- Les hypothèses sont les suivantes:}

Prix de vente du foie gras

rendu en France

Coût de transport avion, emballage compris

Coût emballage et expé-

dition par foie

Poids moyen d'une oie à

l'abattage

Prix de vente de l'oie

Poids moyen des foies

gras

Poids d'une foie emballé
$480 \mathrm{Fmg} / \mathrm{Kg}$

220 Fmg

$7 \mathrm{Kg}$

$200 \mathrm{Fmg} / \mathrm{Kg}$

$0,700 \mathrm{Kg}$

$1,00 \mathrm{Kg}$
$3.700 \mathrm{Fmg} / \mathrm{Kg}$

- Calcul du revenu pour l'oie gavée

\section{Dépenses}

Prix de revient de l'oie gavée

Coût de transport de $0,700 \mathrm{Kg}$

de foie gras

$$
\text { Total : } \frac{700}{2.809}
$$

Recettes

$0,700 \mathrm{Kg}$ de foie gras

Carcasse

3.990

Revenu

$$
3.990-2.809=1.181 \mathrm{Fmg}
$$

Compte tenu d'un certain caractère hypothé- tique des chiffres ci-dessus, on n'a pas fait les calculs des coefficients de rentabilité économique et financière.

\section{Commentaire}

L'analyse financière par phases successives de la production des oies jusqu'au stade ultime du gavage peut donner un aperçu des conditions économiques pour lesquelles l'une ou l'autre phase de la production serait susceptible de s'insérer dans un cadre paysannal.

La phase du gavage et de prélèvement des foies est certainement la plus délicate à mener à bien. De plus elle nécessite l'usage d'un congélateur. Pour ces raisons, dont la première serait seule suffisante, on ne peut pas envisager de la confier à l'exploitant malgache.

La phase d'élevage et de reproduction d'un troupeau d'oies adultes ne présente guère ces difficultés, car l'oie est un animal des plus rustiques. Bien que nous ne disposions pas actuellement des performances d'oies élevées suivant les techniques rurales classiques, on peut avancer que l'oie des Landes ne supporterait probablement pas plus mal que l'oie indigène ce mode de vie. Sans que nous puissions également rien chiffrer, on peut penser cependant que le coût d'alimentation serait très réduit. On pourrait imaginer alors que le paysan vende les œufs d'oies dès qu'ils sont pondus à un Centre d'appui technique ou à une coopérative équipés d'incubateurs et d'éleveuses. Le prix de l'œuf pourrait être fixé à un niveau qui rémunèrerait largement les soins apportés par l'éleveur.

Cependant cette participation rurale serait manifestement sujette à de nombreux aléas dus principalement à l'omniprésence d'oies malgaches dont il a été montré qu'elles ne sont pas susceptibles de fournir, pas plus que les oies métisses, des foies convenables. Nous ne voyons pas résolu ce problème de contrôle, et pour cette raison, la présente idée ne peut être poursuivie.

Finalement c'est la phase d'élevage des oisons jusqu'à 4 ou 5 mois qui poserait le moins de problèmes techniques, les paysans ayant l'habitude de cette pratique. Si le pourcentage du travail dans les charges totales reste un des plus faibles durant cette période, on peut pen- 
ser aussi qu'en milieu rural, les charges de nourriture seraient très diminuées. En particulier le bas-fond naturel remplacerait les prairies temporaires.

Néanmoins, pour envisager cette hypothèse, il faut songer aux difficultés de gestion et au besoin d'encadrement qui résulteraient de l'intégration de cette phase dans un secteur paysannal.

Le rachat des oisons âgés de 4 à 5 mois va en outre être soumis aux conditions du marché de l'oie qui sont les suivantes: l'oison malgache de quelques semaines se vend environ $200 \mathrm{Fmg}$, l'oie malgache adulte aux environs de $1.000 \mathrm{Fmg}$, laissant à l'éleveur une différence de 800 Fmg. L'éleveur qui achèterait un oison des Landes le paierait par contre au moins 660 Fmg à un mois et, pour réaliser le même bénéfice que sur une oie malgache, devrait la revendre à un poids d'environ $6 \mathrm{~kg}$ à $1.500 \mathrm{Fmg}$, prix qui est supérieur au coût de l'oison de quatre mois produit par l'unité technique décrite. Dans la mesure où l'organisme gaveur dispose d'une marge suffisante il pourrait absorber cette différence, l'alternative étant que l'éleveur consente à ne pas faire un bénéfice par oie des Landes aussi important que par oie malgache. Le comportement des parties en présence ne saurait être connu a priori.

L'ensemble de ces remarques fait qu'il semble préférable à l'heure actuelle de n'envisager la production d'oies destinées à être gavées que dans un cadre extra-rural.

L'étude prévisionnelle que nous avons faite dans cette optique se prête à quelques réflexions.

Un certain nombre d'options d'ordre technique ont été choisies de façon assez arbitraire et mériteraient d'être mises en balance dans une étude plus approfondie. Par exemple, on pourrait envisager l'élevage des reproducteurs et des oisons sur caillebotis plutôt que sur aire paillée.

Néanmoins, nous avons opté pour la mécanisation de certains ateliers tels que l'incubation et le gavage. Ce choix d'importance mérite une justification.

En ce qui concerne l'incubation, une étude préliminaire nous a montré qu'un incubateur automatique permettait d'abaisser nettement le coût de l'oisillon d'un jour, principalement en réduisant la main-d'œuvre.

Ce fut encore plus éloquent pour le gavage. Avec un gavoir à main, un gaveur ne peut s'occuper que de 15 oies à la fois, ce qui représente 90 oies pour une période de gavage de six mois. Si le gavage à main avait été retenu, il aurait fallu prévoir une équipe de 23 gaveurs. Ce seul chiffre suffit à montrer, ce qu'une étude préliminaire avait nettement souligné, la nécessité d'un gavage avec appareil à haut rendement.

De telles méthodes de production semblent assurer une compétitivité solide dans la production de foies gras.

\section{ANNEXE}

Couverture de tôles $800 \mathrm{Fmg} / \mathrm{m}^{2}$

Aire bétonnée de $5,5 \mathrm{~cm}$ d'épais. $500 \mathrm{Fmg} / \mathrm{m}^{2}$ Grillage simple torsion $(8 \times 8)$

$$
\text { largeur } 2,5 \mathrm{~m}
$$

Grillage simple torsion $(8 \times 8)$

$245 \mathrm{Fmg} / \mathrm{m}$

$$
\text { largeur } 1,25 \mathrm{~m}
$$

Prix du kWh sur courant

force

$150 \mathrm{Fmg} / \mathrm{m}$

Prix d'un bâtiment en dur

14 Fmg

Prix du maïs

Prix de l'engrais

$5.000 \mathrm{Fmg} / \mathrm{m}^{2}$ couvert

$10 \mathrm{Fmg} / \mathrm{Kg}$

$35 \mathrm{Fmg} / \mathrm{Kg}$

\section{Main-d'auvre}

Le Centre National Avicole est situé en zone de Salaire III

Rémunération mensuelle

$(200 \mathrm{~h} /$ mois $)$

Manœuvre sans qualification 4.320 Fmg

Ouvrier agricole spécialisé (OS1) 5.760

Ouvrier agricole spécialisé (OS2) 7.200

Charges sociales, allocations fami-

liales, assurance accident, 7,5 p. 100

de la rémunération.

\section{Amortissement} des investissements

- Amort. sur 4 ans au taux d'intérêt de $6 \mathrm{p}$. 100

Prairies temporaires Coût/ha
Facteur d'amortissement 0,2886 35.700 Fmg 
- Amort. sur 5 ans au taux d'intérêt de $5 \mathrm{p}$. 100

Clôtures grillagées

\section{Amortissement}

des investissements

Facteur

d'amortissement

- Amort. sur 10 ans au taux d'intérêt de $4 \mathrm{p}$. 100

Réfrigérateur -

Coût unitaire

Congélateur -

Coût unitaire

86.000 Fmg

Gavoir électrique -

145,000

Coût unitaire

40.000

Eleveuse -

Coût unitaire

31.000

Hache-paille -

Coût unitaire

100.000

- Amort. sur 15 ans au taux d'intérêt de 4 p. 100

Incubateur -

Coût unitaire

300.000

- Amort. sur 20 ans au taux d'intérêt de $4 \mathrm{p}$. 100

Hangar de ponte

Bâtiment d'élevage des oisons de un à quatre mois

Oisonnière - Coût $/ \mathrm{m}^{2}$

Bâtiment de gavage -

Coût $/ \mathrm{m}^{2}$

5.000

Loges de gavage -

Coût unitaire

5.000

750

Travail à l'entreprise

Coût d'implantation

d'un hectare de prair̂ie temporaire :

\begin{tabular}{lr} 
Labour & 5.000 \\
Préparation du sol & 2.500 \\
Graines $6 \times 1200$ & 7.200 \\
Engrais & 21.000 \\
\hline & 35.700 \\
\hline
\end{tabular}

\section{CONCLUSIONS}

En définitive, il résulte de l'expérience acquise à ce jour que l'élevage de l'oie landaise à Madagascar présente un réel intérêt.
S'il existe des difficultés relatives à l'incubation et éventuellement à la période de trois semaines de l'élevage des oisillons, il reste toutefois que l'élevage de l'oison, l'entretien d'un troupeau de mères, et même le gavage sont à la portée de quiconque s'y donne sérieusement.

Aucune difficulté majeure d'ordre pathologique ou alimentaire n'est apparue; un facteur essentiel de la réussite est, là aussi sans doute, la bonne qualité de la verdure distribuée; les locaux d'élevage sont sommaires, la conduite du troupeau et le gavage faciles; finalement, moyennant le respect de quelques règles fondamentales, les performances sont proches de celles observées sous les climats tempérés.

D'ailleurs, quelques animaux, purs et métis, ont déjà été cédés à des particuliers auxquels ils donnent pleine satisfaction malgré des soins peu attentifs.

Il apparaît en outre que la chaleur et l'humidité, telles du moins qu'on les rencontre sur 0,0899 les plateaux malgaches (Tananarive et MoyenOuest) n'ont pas d'influence néfaste sur le gavage.

La longueur particulièrement importante de la saison de ponte et son décalage par rapport 0,0736 aux périodes de l'hémisphère Nord sont particulièrement intéressants dans l'optique du gavage. Ces caractéristiques permettront en effet d'alimenter en quasi-permanence en produits frais les conserveries.

Compte tenu du classement qualitatif des foies en fonction de leur poids, il n'est pas nécessaire de rechercher de très gros foies; ceux-ci sont beaucoup plus coûteux, sous-classés à la vente et sont plus fragiles à l'extraction.

Il convient donc d'obtenir des foies moyens $(600-900 \mathrm{~g})$ et le plus rapidement possible (économie de main-d'œuvre, économie de maïs, rapidité de rotation des lots). Pour cela, il semble indispensable de pousser le gavage, mais en restant dans des conditions sanitaires convenables: claustration étroite, obscurité, humidité, chaleur, trois gavages par jour avec deux passages chaque fois, ceci au moins pendant la première semaine; pendant les derniers jours, il est possible éventuellement de soulager et de prolonger l'animal par douchage et séjour sur caillebotis en local ventilé. Enfin, il est absolument nécessaire de n'utiliser que du maïs à peine ébouillanté; sinon cela reviendrait à gaver avec de l'eau. 


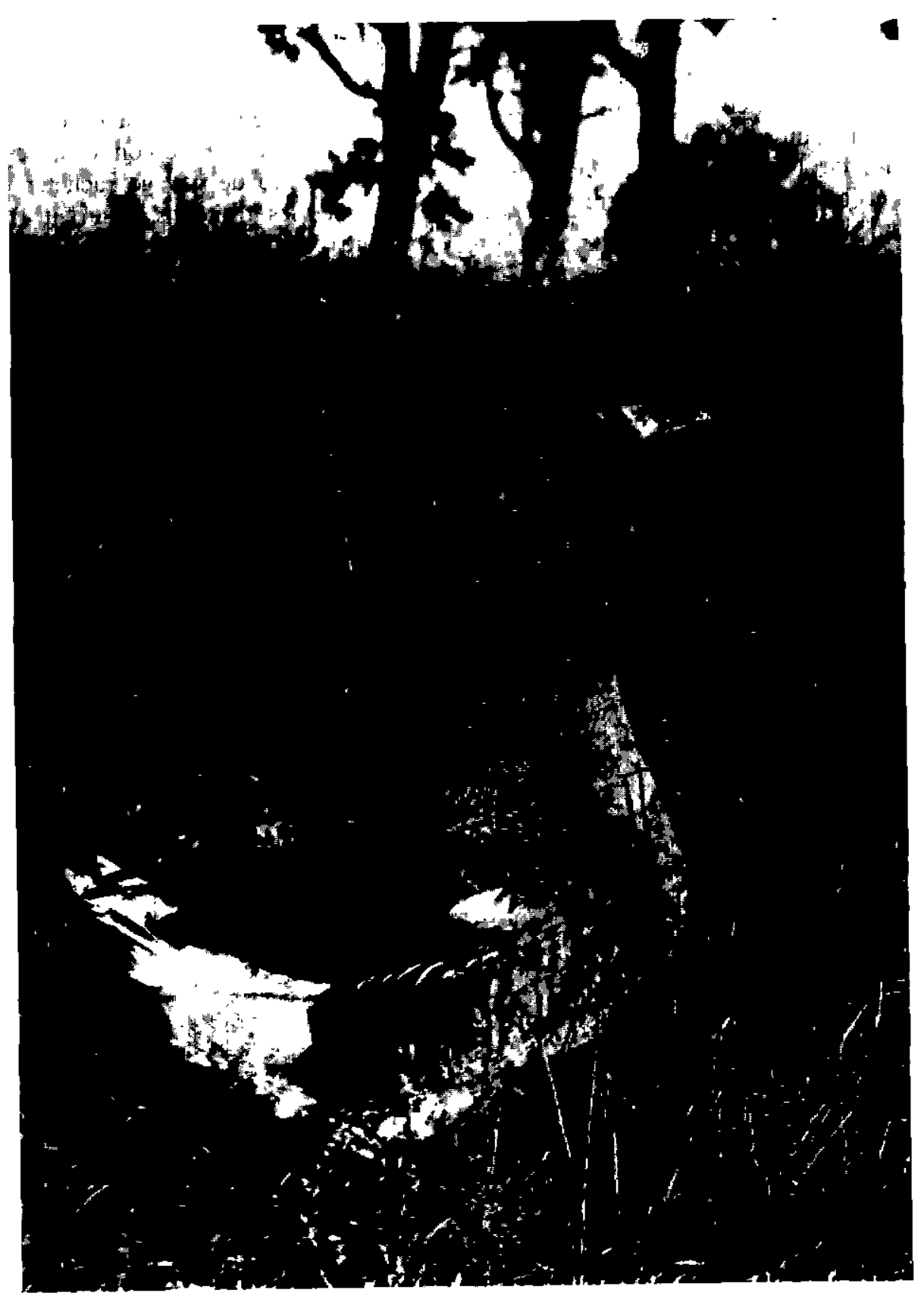

no 1. Oie des Landes née et élevée à Madagascar. (Pâturage de Chloris gayana). 
Il faut aussi apporter à l'abattage les plus grands soins, notamment rechercher une saignée complète car les foics mal saignés sont dépréciés. Les foies obtenus dans ces conditions sont d'excellente qualité, et peuvent être exportés, soit réfrigérés, soit congelés.

La qualité des foies d'oies métisses est honorable, toutefois leur coût de production abstraction faite du coût de fabrication ou du prix d'achat de l'oie - en est relativement supérieur et beaucoup devront être déclassés du fait d'un poids trop faible.

Compte tenu de la bonne prolificité observée des oies des Landes, il ne semble pas qu'on doive s'attarder à produire des animaux métis qui rentabiliseront bien moins l'opération.

Le gavage des oies malgaches n'a donné aucun résultat du point de vue foie gras, et des résultats mauvais à propos de l'engraíssement des carcasses.

Aussi, au vu de l'ensemble des résultats, il semble que l'élevage de l'oie landaise pure puisse être dès à présent proposé à la vulgarisation. On peut suggérer que le Centre National Avicole, déjà pourvu d'une infrastructure moderne, prenne en charge la production d'oisillons qui pourraient être confiés à des éleveurs. Puis un Centre de gavage rachèterait les oies à gaver et les traiterait, assurant l'expédition aérienne des foies gras congelés et commercialisant les carcasses.

Mais ce schéma n'est pas le seul possible; toute société d'Etat ou privée pourrait s'équiper pour la mise en ceuvre de la chaîne complète des opérations, sans que cela nécessite des investissements extrêmement lourds.

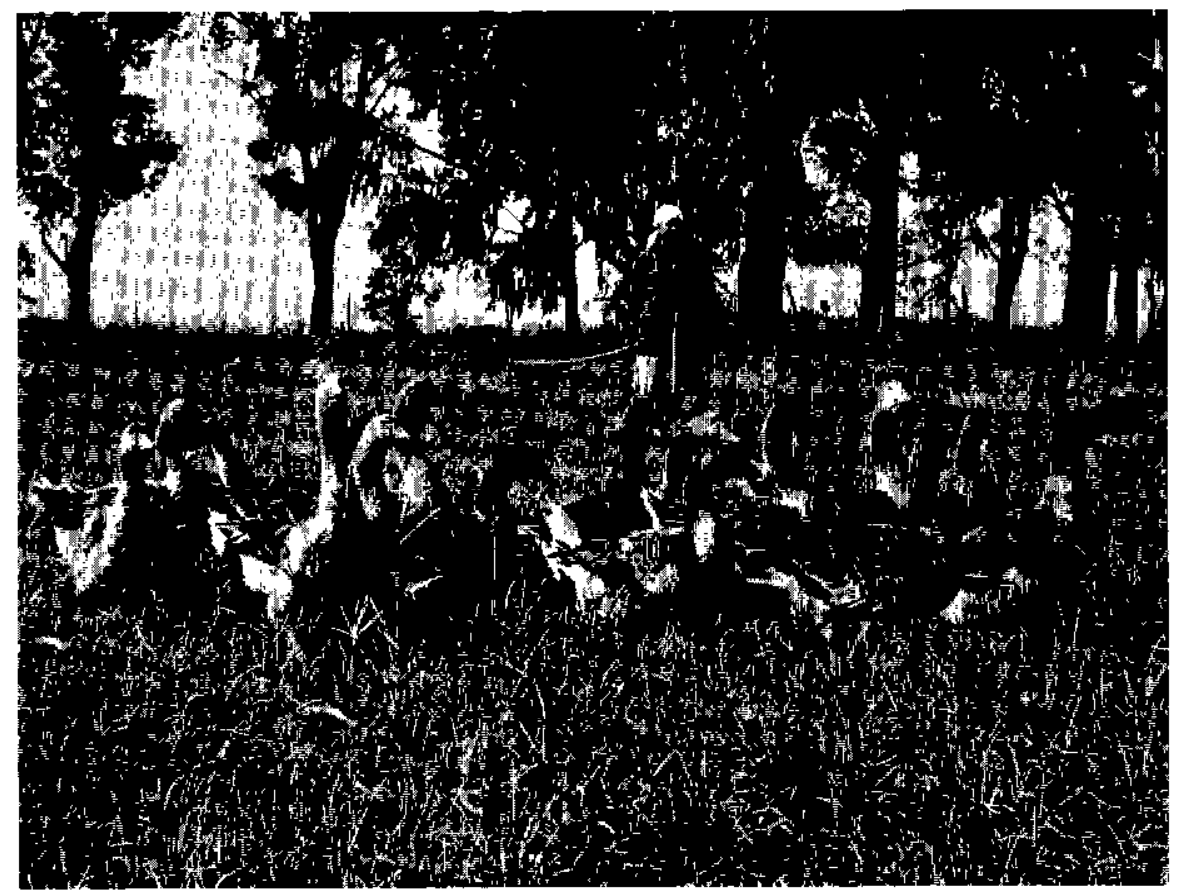

$n^{\circ} 2$. Gardeuse d'oies et son troupeau. 


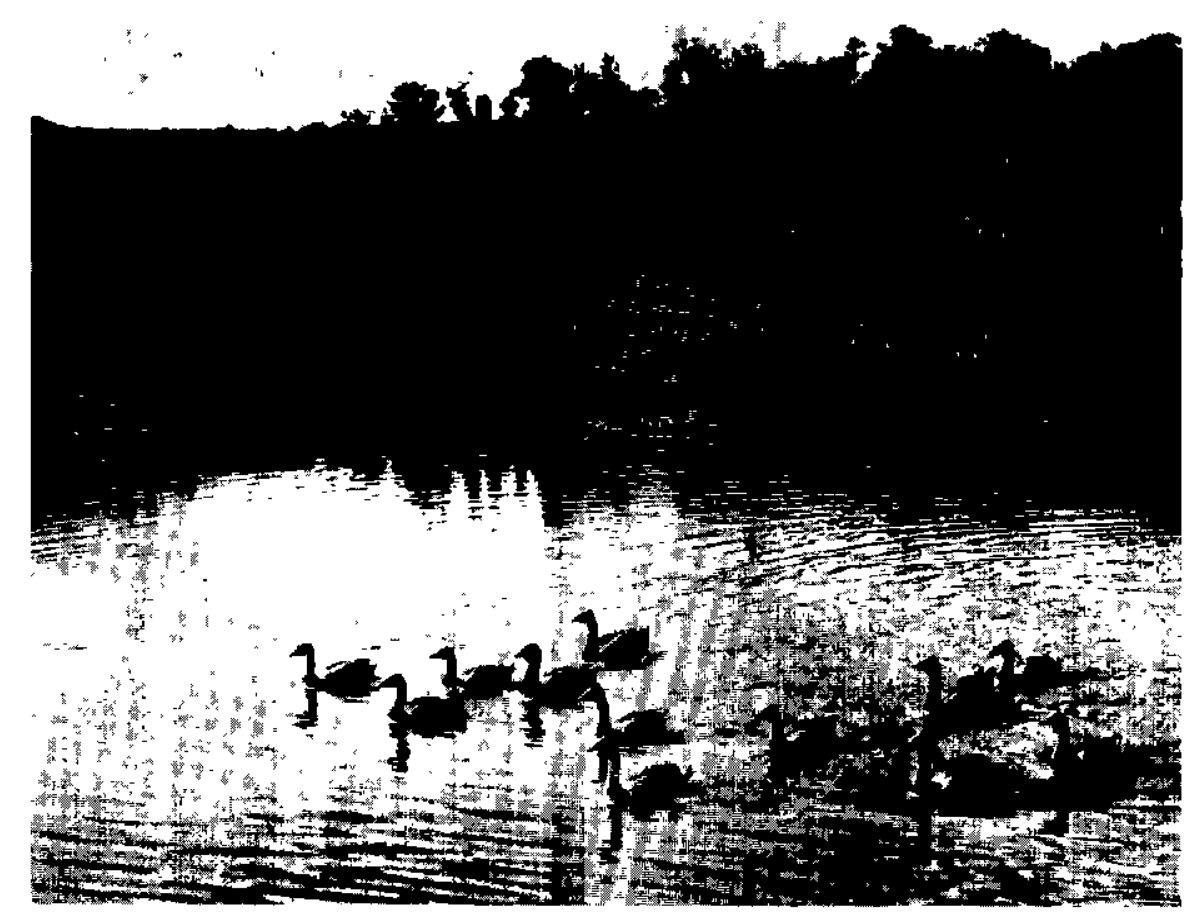

n" 3. Elevage mixte d'oies des Landes et métisses de type paysannal.

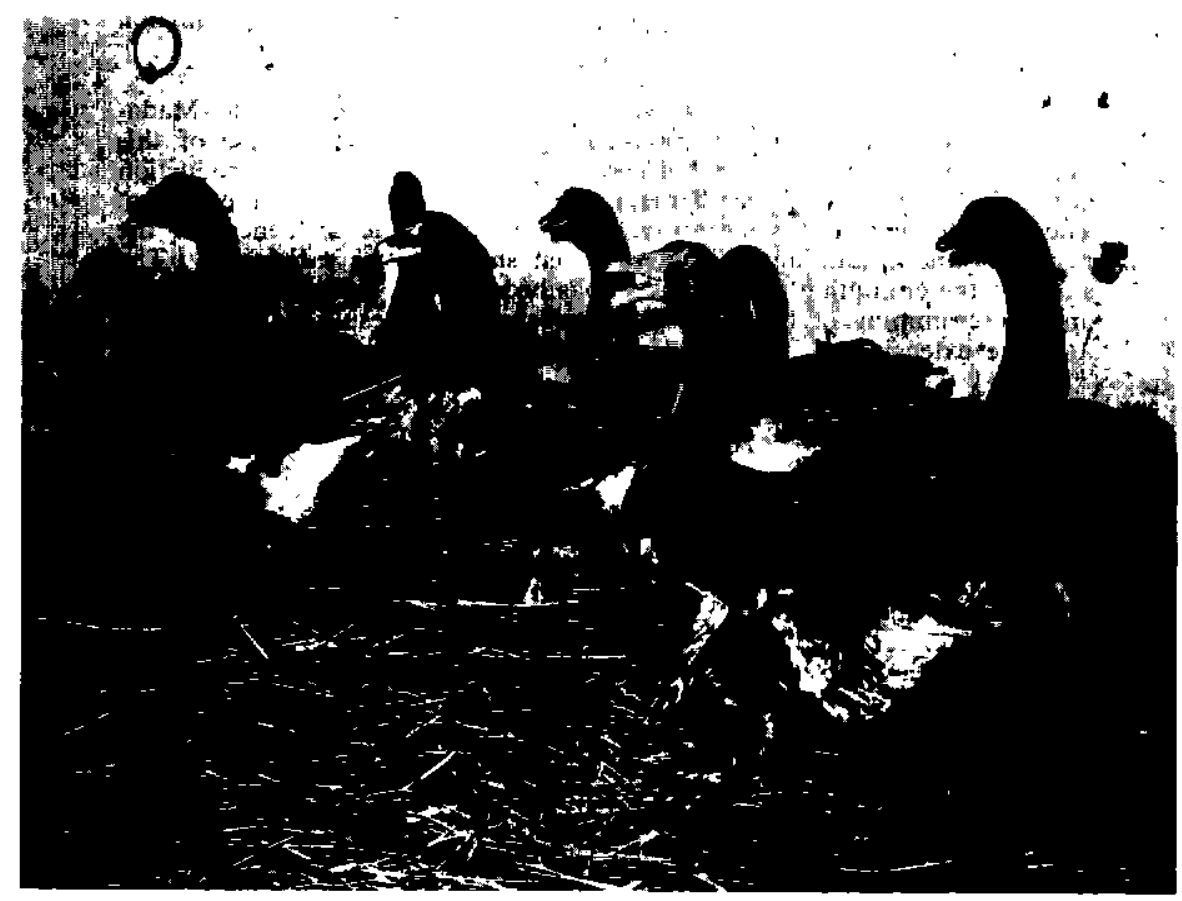

no 4. Oies des Landes en cours de gavage. 


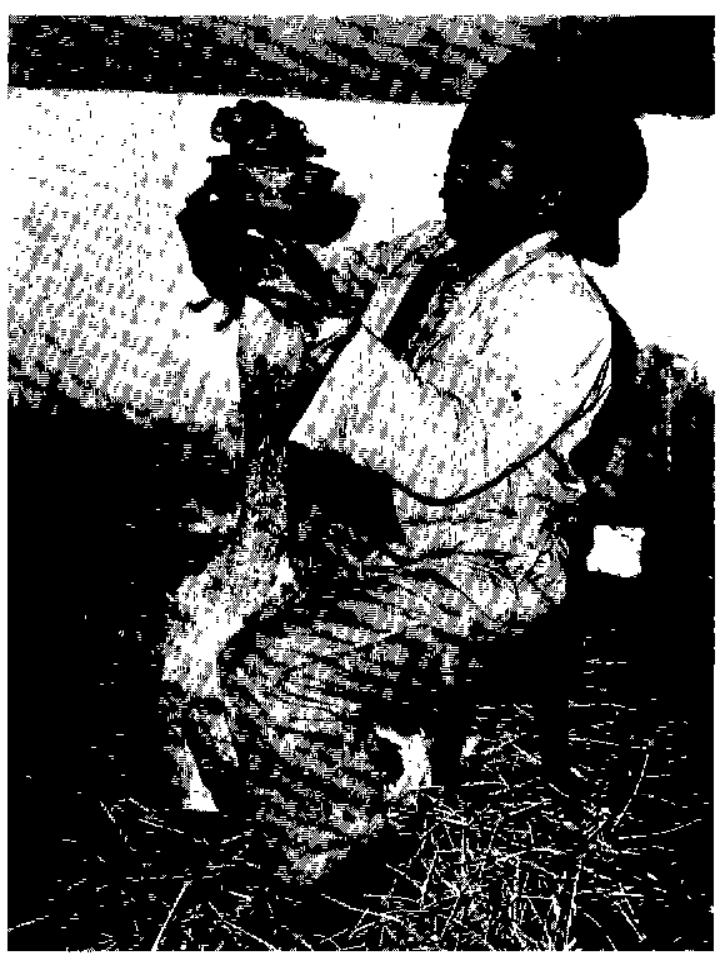

$n^{0}$ 5. Gavage à la main.

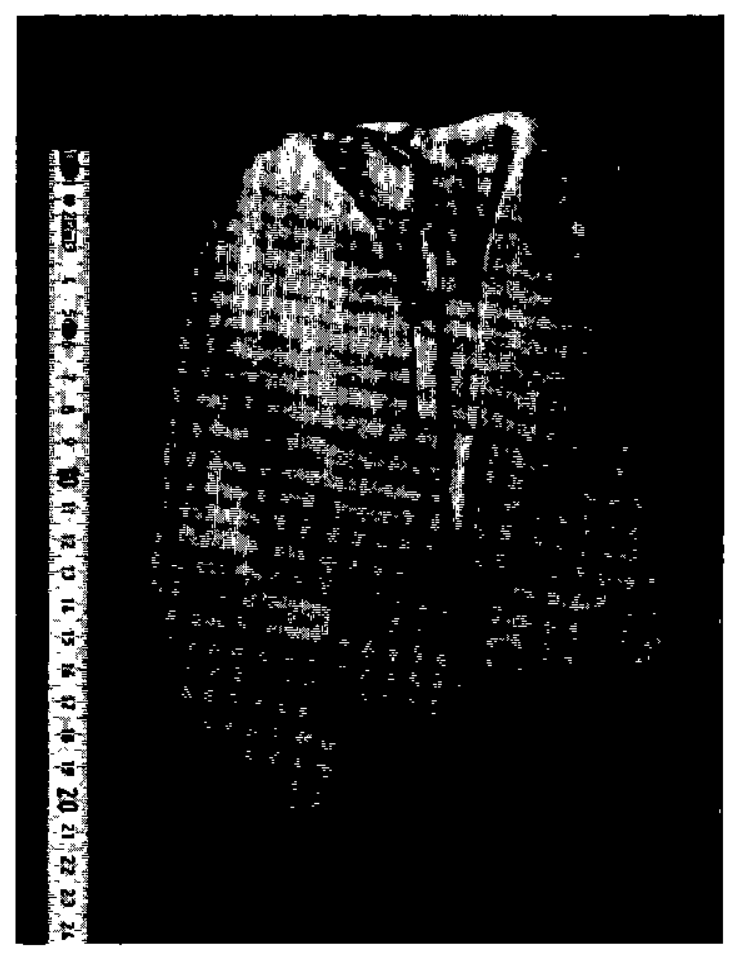

n"6. Un beau foie de 1.000 grammes,

\section{SUMMARY}

Note on Landes goose breeding with a trial of « foies gras" production in Madagascar

The breed of goose of the Landes has been introduced into Madagascar with a view to producing «foie gras». The performance of this breed is identical to that observed in a temperate climate. The reproduction period is longer and staggered. within, however, the limits of the season. The climate does not seem to have an unfavourable influence on the results of the cramming which have been shown to be satisfactory. The study of the cramming of local geese has been carried out without success, and the cramming of half-bred geese gave medium results.

The extension of breeding of Landes geese has been encouraged.

\section{RESUMEN}

Nota sobre una crianza de gansos de las Landes con ensayos de producción de «foie gras " en Madagascar

Se introdujó la raza de gansos de las Landes para la producción de " foie gras $»$.

Los rendimientos de ésta crianza son iguales con los observados bajo un clima temprano. El periodo de reproducción es más largo, decalado pero en los límites de la estación. El clima no parece tener una influencia desfavorable sobre los resultados de la cebadura que son muy satisfactorios. Se hizo el estudio de la cebadura de los gansos del país sin exito y el de los gansos mestizos dió resultados medios.

Se propone la vulgarización del ganso de las Landes. 


\section{BIBLIOGRAPHIE}

Documents de travail. Congrès de loie. JOUY-ENJOSAS. 1967.

MONACHON (G), «Queiques réflexions sur l'élevage et l'habitat des oies ", Rev. Elev., 1965, 20, 11, 105-121.

L'oie. Rev aricole, 1963, 73, 12 (n's spécial).

PAVAUX (Cl.), FAYET (G.), «Contribution à l'étude de la croissance de l'oie, de la 1re à la 13 e semaine », Rev. Méd. Vét., 1966, 117, 5, 577-92.
Rapport présenté aux Journées scientifiques sur la physsologie, la pathologie, la chimie et la cytologie des foies gras, 24-26 mars 1953. Asn. Nutr. Alim., 1953, 7, 6.

SZUMOWSKI (P.), IVACHKIEVITCH (N.), «La production du foie gras d'oies âgées de moins de cinq mois ", Rev. Eler., 1966, 21, 10. 129-130.

VUATRIN (B.), «La production du foie gras », Paris, J.B. Baillière, 1965 . 